Review Article

\title{
Epigenetic versus Genetic Deregulation of the KEAP1/NRF2 Axis in Solid Tumors: Focus on Methylation and Noncoding RNAs
}

\author{
F. P. Fabrizio $\mathbb{D}$, A. Sparaneo, D. Trombetta, and L. A. Muscarella $\mathbb{D}$ \\ Laboratory of Oncology, IRCCS Casa Sollievo della Sofferenza Hospital, San Giovanni Rotondo, Italy \\ Correspondence should be addressed to F. P. Fabrizio; federico_fabrizio@hotmail.it
}

Received 25 August 2017; Revised 20 November 2017; Accepted 4 December 2017; Published 13 March 2018

Academic Editor: Bhairab N. Singh

Copyright (c) 2018 F. P. Fabrizio et al. This is an open access article distributed under the Creative Commons Attribution License, which permits unrestricted use, distribution, and reproduction in any medium, provided the original work is properly cited.

\begin{abstract}
Oxidative and electrophilic changes in cells are mainly coordinated by the KEAP1/NRF2 (Kelch-like erythroid-derived cap-n-collar homology- (ECH-) associated protein-1/nuclear factor (erythroid-derived 2)-like 2) axis. The physical interaction between these two proteins promotes the expression of several antioxidant defense genes in response to exogenous and endogenous insults. Recent studies demonstrated that KEAP1/NRF2 axis dysfunction is also strongly related to tumor progression and chemo- and radiotherapy resistance of cancer cells. In solid tumors, the KEAP1/NRF2 system is constitutively activated by the loss of KEAP1 or gain of NFE2L2 functions that leads to its nuclear accumulation and enhances the transcription of many cytoprotective genes. In addition to point mutations, epigenetic abnormalities, as aberrant promoter methylation, and microRNA (miRNA) and long noncoding RNA (lncRNA) deregulation were reported as emerging mechanisms of KEAP1/NRF2 axis modulation. This review will summarize the current knowledge about the epigenetic mechanisms that deregulate the KEAP1/NRF2 cascade in solid tumors and their potential usefulness as prognostic and predictive molecular markers.
\end{abstract}

\section{Introduction}

Oncogenes and tumor suppressor genes are deregulated in cancer and modify their expression through heterogeneous genetic and epigenetic modifications. All these alterations exert their effects on several cellular processes in which transient modifications of redox balance might occur, such as cell cycle and apoptosis. These transient cellular changes are mainly coordinated by the KEAP1/NRF2 (Kelch-like erythroid-derived cap-n-collar homology- (ECH-) associated protein-1/nuclear factor (erythroid-derived 2)-like 2) signaling pathway [1]. NRF2 is a transcription factor that acts as a master modulator of cellular defense against toxic and oxidative damage, mitochondrial physiology, differentiation, and stem cell maintenance [2-4]. In normal cell conditions, the NRF2 negative regulator KEAP1 forms an ubiquitin ligase complex with cullin 3 (CUL3) and ring-box 1 (RBX1) and targets NRF2 for proteolysis. Upon stress exposure, a specific pattern of KEAP1 cysteine modification arises. By consequence, the KEAP1 releases NRF2 which translocates into the nucleus, where it forms a heterodimeric complex with the small MAF proteins. This complex recognizes the enhancer sequences known as antioxidant response elements (AREs) located in the cytoprotective genes and activates their transcription [5]. Additionally, NRF2 can be subjected to a KEAP1-independent transcriptional and posttranslational regulation, with a consequent alteration of its cellular localization, protein degradation, and DNAbinding capability [6].

Deregulation of the KEAP1/NRF2 axis is actually considered a hallmark in cancer cells, since KEAP1 and NRF2 can modulate oncogenesis, cell proliferation, apoptosis, and tumor cell growth [7].

A decreased levels of KEAP1 protein were firstly reported to be linked to poor outcome in NSCLC patients treated with platinum-based neoadjuvant regimen and in the NSCLC group of patients with lymph node metastases $[8,9]$.

Actually, the main molecular events that lead to NRF2 abnormal nuclear accumulation in solid tumors can be generally divided into genetic and epigenetic alterations. The final effect is in any case the disruption of protein-protein interaction of the KEAP1/NRF2 crosstalk and its imbalance 
in expression levels, with a consequent upregulation of cellular detoxifying proteins.

Genetic alterations were the first reported mechanisms of KEAP1/NRF2 axis deregulation. Biallelic changes of the tumor suppressor KEAP1 gene (by point mutations and loss of heterozygosity) were described for the first time in NSCLC [10] and, immediately after, in other several malignancies together with NFE2L2 mutations, the gene that codifies for the NRF2 protein [11]. More recently, the discovery of hypermethylation of the KEAP1 promoter and noncoding RNAs linked to cell-detoxifying network added a new important dimension in the complex regulation of the KEAP1/ NRF2 system (Figure 1) [12].

This minireview describes the recent updates about the deregulation mechanisms of the KEAP1/NRF2 pathway, with a particular focus on the epigenetic modulation of KEAP1 and NFE2L2 and their cellular significance and potential impact on cancer patient management.

\section{The Genetic Deregulation of Keap1/Nrf2 Signaling and Its Translational Impact in Solid Tumors}

Among the genetic lesions that affect the KEAP1/NRF2 activity, point mutations are the most frequently investigated ones in solid tumors. They commonly occur in the exonic regions that codify for the KEAP1 and NRF2 interaction sites (the Kelch/DGR domain of KEAP1 and the Neh2 domain of NRF2) and induce a general failure of the ubiquitination process led by the KEAP1 (Figure 2).

By consequence, the NRF2 escapes from proteasomal degradation and increases the ARE-target gene expression with an enhancement of the antioxidant defense system and chemo- and radioresistance of cancer cells [13, 14]. Moreover, since the KEAP1 is able to negatively modulate the BCL-2 and p62 degradation, the KEAP1 point mutations also lead to an accumulation of these proteins with a general deregulation of apoptosis, autophagy, and inflammation [15, 16].

Loss-of-function mutations of the human KEAP1 gene have been firstly reported in NSCLC with a prevalence of about $20-25 \%$. These mutations were frequently observed in the lung papillary subtype and in TTF- 1 negative lung adenocarcinoma $[10,17,18]$. Moreover, KEAP1 point mutations were identified in several human cancers such as gastric (11.1\%), liver (2-8\%), colorectal (7.8\%), prostate (1.3\%), gallbladder $(30.7 \%)$, ovarian (37\%), glioma (1.7\%), head and neck (42\%), and clear renal cell carcinoma (4.7\%) [19-29]. More recently, Fernandez-Cuesta et al. and Derks reported KEAP1 genetic alterations as a new uncovered molecular hallmark of LCNEC (lung cancer neuroendocrine) with adenocarcinoma-like features $[30,31]$. This last finding was also confirmed by a different group which reported a prevalence of KEAP1-NFE2L2 (31\%) alterations in tumors with high neuroendocrine gene expression, mainly cooccurrent with gene mutations $[4,32,33]$.

Gain-of-function NFE2L2 mutations are generally mutually exclusive with respect to KEAP1 mutations and are frequently located into the DLG or ETGE motifs. NFE2L2 point mutations were identified in several tumors with squamous histological features, such as esophageal, skin, lung, and laryngeal carcinomas. An increased NFE2L2 gene copy number was also described in lung squamous cell carcinoma. An increased NFE2L2 gene copy number was also described in lung squamous cell carcinoma. All of these mutations are generally missense changes that interfere with the KEAP1 ability to bind to NRF2, thus inducing an escape of NRF2 degradation without changing its gene functionality [34-37]. Somatic lesions linked to KEAP1/NRF2 axis deregulation were also reported in the CUL3 gene, the component of the E3 ligase complex KEAP1/CUL3/RBX1 that marks NRF2 for proteasomal degradation $[7,38]$. Mutations in CUL3, together with those in NFE2L2, are frequent in hereditary type 2 papillary renal cell carcinoma (PRCC2). In squamous carcinoma of the head and neck, somatic lesions of CUL3/NFE2L2/KEAP1 have a prevalence of $64 \%$ and were associated with a lower patient overall survival (Tables 1 and 2) [25, 39-41].

Dysfunction of the KEAP1/NRF2 axis by genetic mutations is gradually becoming a milestone to understand cancer development, progression, and resistance to conventional and biological treatments [42]. It is now well known that loss-of-function mutations of the KEAP1 gene or gain-offunction mutations in NFE2L2 enhance the resistance of cancer cells to anticancer drugs, such as etoposide and carboplatin, and it is associated with poor outcome of platinum-based advanced NSCLC patients [43]. Nuclear accumulation of NRF2 was also correlated with a poor survival of lung SqCC and pancreatic adenocarcinomas and a worse progression free survival (PFS) in patients treated with surgery only [44-47]. Aberrant NRF2 activation due to KEAP1 alterations is also reported as one of the molecular mechanisms of chemoresistance of gallbladder cancer under 5-FU-based regimen and of colorectal cancer under demethylase and methyltransferase treatments [20, 48, 49].

Jeong and coworkers suggested a new role for KEAP1 and NFE2L2 mutations in radiotherapy resistance of NSCLC patients and in identifying patients who might benefit from radiation dose escalation [50]. Knockdown experiments reported that radiochemosensitization was led by CDK20 that competes with NRF2 for KEAP1 binding and induces nuclear translocation of NRF2 and the enhancement of its transcriptional activity. This ultimately results in proliferation defects and provides new insights into the cellular response to NRF2-mediated DNA damage [51].

An interesting link between the KEAP1/NRF2 axis and target therapies was recently reported. Cell proliferation in cancer was demonstrated to be cross-regulated by KEAP1/ NRF2 and EGFR signaling. Moreover, cells expressing oncogenic allele of KRAS are able to activate NRF2 via the MAPK pathway in mouse embryonic fibroblasts $[52,53]$. In the same way, the loss of KEAP1 by the CRISPR-Cas9 system cooperates with the tumor mutational landscape in modulating the response to BRAF, MEK, EGFR, and ALK inhibition and in allowing cancer cells to increase their ability to resist to treatments and proliferate [54]. KRAS activity confers in NSCLC chemoresistance also by upregulating NRF2 through the link with TPA response element (TRE) located in exon 1 of the NFE2L2. In the same context of resistance to target therapy, it is possible that NFE2L2 mutations can contribute to 


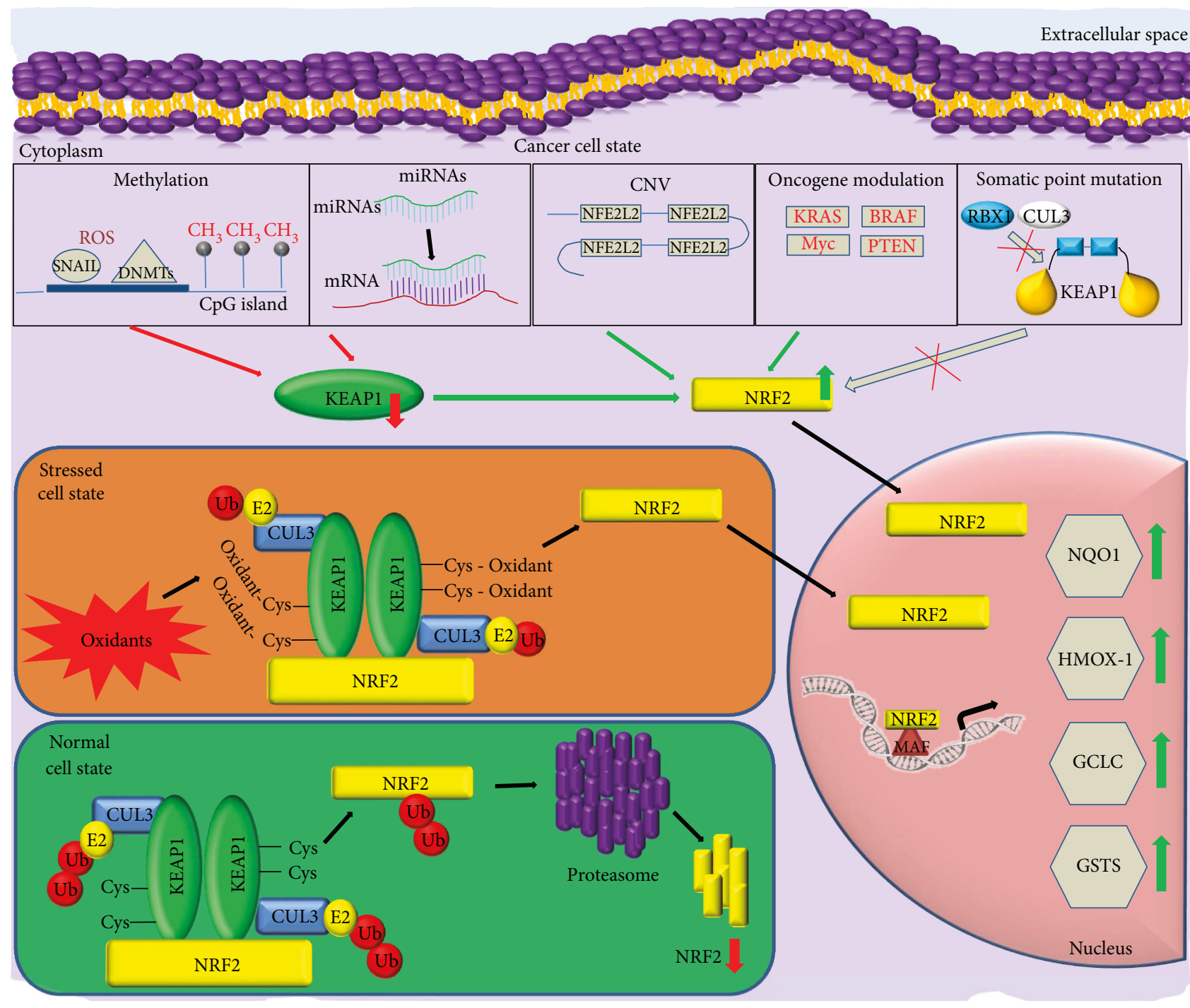

FIGURE 1: Overview of the main genetic and epigenetic modifications that lead to KEAP1-NFE2L2 impairment and constitutive NRF2 nuclear accumulation in cancer cells. NFE2L2 gene copy number variations (CNV), oncogene activity (KRAS, BRAF, MYC, and PTEN), DNA promoter methylation, and miRNAs contribute in a synergic manner to increase cancerous NRF2 activity as a result of reduction of KEAP1 mRNA or increase of NRF2 mRNA levels and/or protein expression. By contrast, somatic point gain-of-function mutations in NFE2L2 or in loss-of-function in KEAP1 promote the disruption of the interaction between KEAP1 and NRF2 and lead the increase of NRF2 protein quantity which translocates into the nucleus.

survival under crizotinib treatment and can allow the cells to acquire additional resistance mutations over time. In line with these hypotheses, Krall et al. recently identified a hotspot mutation in NFE2L2 in a patient with acquired resistance to ALK inhibitors that could exert a synergic effect with a secondary $A L K$ mutation in the resistance to secondgeneration ALK inhibitors [55].

Recent additional studies in this field gave the first hint of the prognostic role of single-nucleotide polymorphisms (SNPs) of the KEAP1 gene in breast cancer without inducing any evident and detectable variations of the protein structure or conformation. More specifically, five tagging SNPs (rs34197572, rs9676881, rs1048290, rs11085735, and rs8113472) located in the KEAP1 were genotyped and appeared to be in allelic linkage disequilibrium (LD) with each other. This finding suggests the existence of a haplotype block at the KEAP1 gene locus that might correlate with specific clinical features of cancer patients [56]. The two SNPs rs9676881 and rs1048290 resulted to be significantly associated with a shorter PFS survival in invasive breast cancer patients. The main hypothesis is that they reside into cell type-specific regulatory elements that modulate the binding capability of critical transcriptional factors, which in turn change target gene expression. Thus, it might explain the observed correlation with the high KEAP1 protein expression levels and the high cytoplasmic localization of NRF2 in breast tissues [57]. The SNP rs1048290 is located in the DGR domain, so it may affect the maintenance of physiological levels of NRF2.

Tumor susceptibility SNPs might be also associated with specific miRNA/lncRNA binding regions $[58,59]$. The SNP rs1048290 was found in LD with the SNP rs9676881, which 


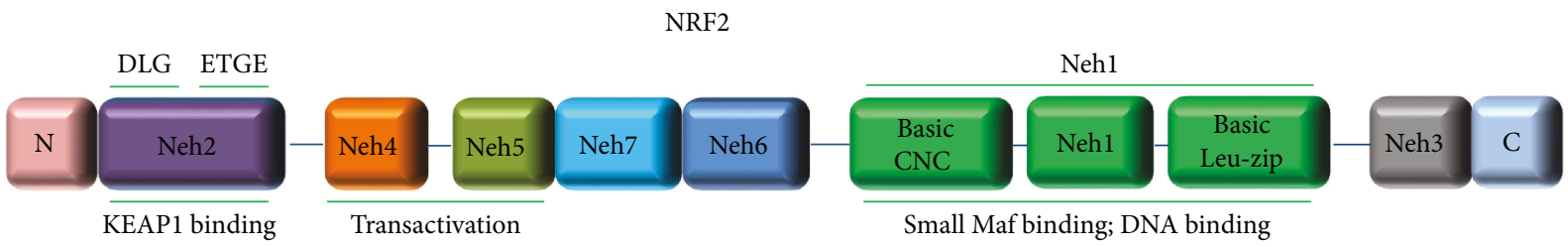

(a)

KEAP1

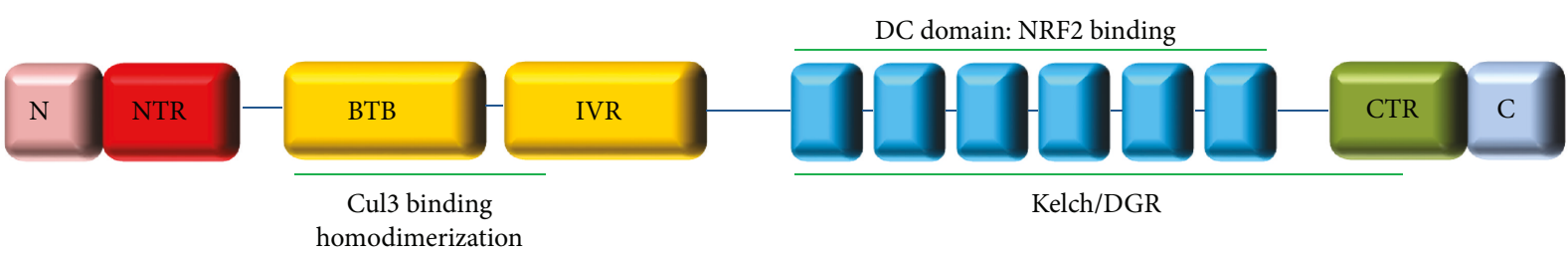

(b)

FIgURE 2: Domain architecture of the NRF2 (a) and KEAP1 (b) proteins. (a) NRF2 protein is divided into seven highly conserved domains, Neh1 to Neh7 (NRF2-ECH homology: Neh). The coordinates of NRF2 protein domains are shown as follows: Neh2 (16-89aa); Neh2 DLG motif (17-32aa), Neh2 ETGE motif (77-82aa), Neh4 (111-134aa), Neh5 (182-209aa), Neh7 (209-316aa), Neh6 (337-394aa), Neh1 (435$568 \mathrm{aa}$ ), and Neh3 (569-605aa). (b) KEAP1 protein contains a number of functional domains including the N-terminal region (NTR; 160aa), broad complex, tramtrack and bric-a-brac (BTB; 61-179aa), the intervening linker domain (IVR; 180-314aa), the double glycine/ Kelch domain harboring six Kelch-repeat domains (315-359aa; 361-410aa; 412-457aa; 459-504aa; 506-551aa; 553-598aa), and the Cterminal region (CTR; 599-624aa).

is located in a putative enhancer region, few bases downstream of the $3^{\prime}$-untraslated region $\left(3^{\prime}\right.$-UTR) of the KEAP1 gene and $410 \mathrm{bp}$ from the miR-200a binding site. By consequence, it is clear to suppose that a LD may exist with these silent variations and the specific miRNA binding site. However, the role of KEAP1 SNPs in predicting patient survival remains controversial. The two SNPs rs9676881 and rs1048290 appeared to be the most interesting ones and resulted to be significantly associated with a shorter PFS survival in both invasive and ER-positive tamoxifen-treated invasive breast cancer patients [60].

\section{The Aberrant Methylation of the Keap1-Nrf2 Axis and Its Translational Impact in Solid Tumors}

Gene promoter hypermethylation at the specific CpGs and chromatin remodeling are two of the main epigenetic events that can modulate gene expression by spatial interfering with the ability to work with the transcriptional machinery.

Epigenetic mechanisms are clearly implicated in the complex regulation of the KEAP1/NRF2 axis and are actually considered the most frequent mechanisms of KEAP1 silencing in solid tumors [61]. The first hypothesis of an epigenetic dysregulation of the KEAP1 gene comes from the intriguing observation that the KEAP1 mutations were not frequent enough to justify alone the frequency of aberrant NRF2 nuclear accumulation reported in lung tumor cells [8]. All the scientific findings on the hypermethylation of the KEAP1 promoter and its effects on the KEAP1/NRF2 pathway are summarized in Table 3. The first report in this field was in human NSCLC and prostate DU-145 cancer cell lines. The promoter $\mathrm{CpGs}$ affected by this phenomenon are grouped into one island located at the $\mathrm{P} 1$ region of KEAP1, near the transcriptional start site [62-64]. The KEAP1 promoter hypermethylation was described in neoplastic tissues of patients affected by glioma, breast cancer (51\%), and primary NSCLC (47\%). In lung cancer, the presence of epigenetic abnormalities in the KEAP1 gene plus its point mutations/ $\mathrm{LOH}$ matched with the prevalence of NRF2 nuclear accumulation in NSCLC tissues and was associated with an increased risk of lung cancer progression in surgically resected patients [65-67]. In clear renal cell carcinoma (ccRCC), the epigenetic modulation of KEAP1 was shown to be the leading mechanism of KEAP1 deregulation (48.6\%), thus supporting a driver role of the KEAP1/NRF2 axis in renal cancer. TCGA (The Cancer Genome Atlas) concomitant data analysis suggested that KEAP1 hypermethylation is able to strongly predict patient survival [68]. In primary breast cancers and preinvasive lesions, an aberrant KEAP1 promoter methylation was seen to be more recurrent in ER-positive, HER2negative than in triple-negative breast cancers and was hypothesized to be a prognostic marker since a higher mortality risk in triple-negative patients was predicted. Moreover, KEAP1 promoter silencing by methylation was also predictive of a lower risk of tumor relapse in patients treated with sequential therapy of anthracyclines and cyclophosphamide followed by taxanes [67]. Gliomas are the second tumor described by our group to have a promoter hypermethylation. In these tumors, it was reported that KEAP1 epigenetic modifications were associated with poor prognosis and contribute to the prediction of the disease progression of patients subjected to radiotherapy and temozolomide treatment [65]. The role of KEAP1/NRF2 in radiation has been also elucidated in A549 lung adenocarcinoma cell line under DNMT inhibitor genistein treatment. The pharmacological demethylation of the KEAP1 CpG promoter 


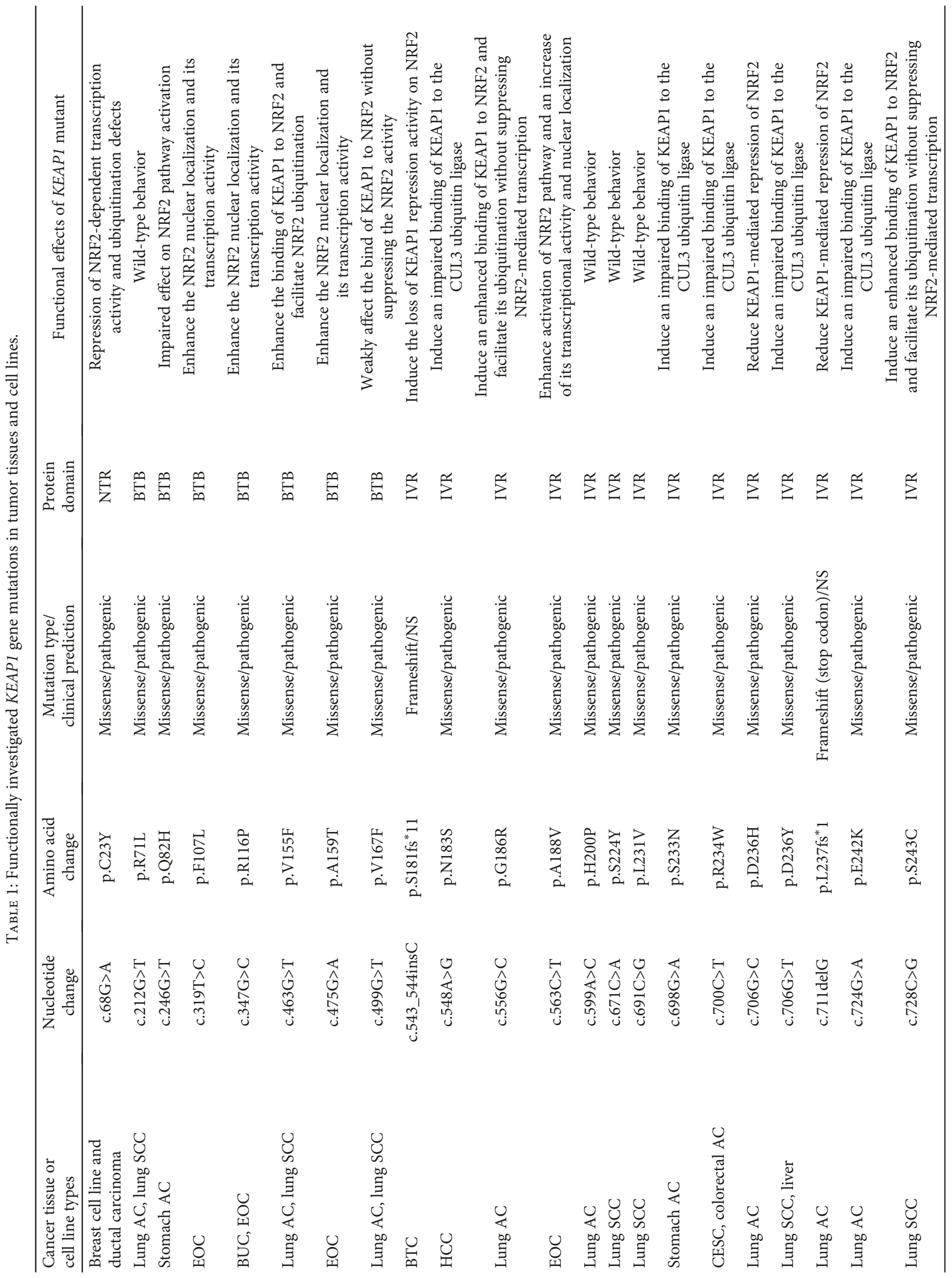




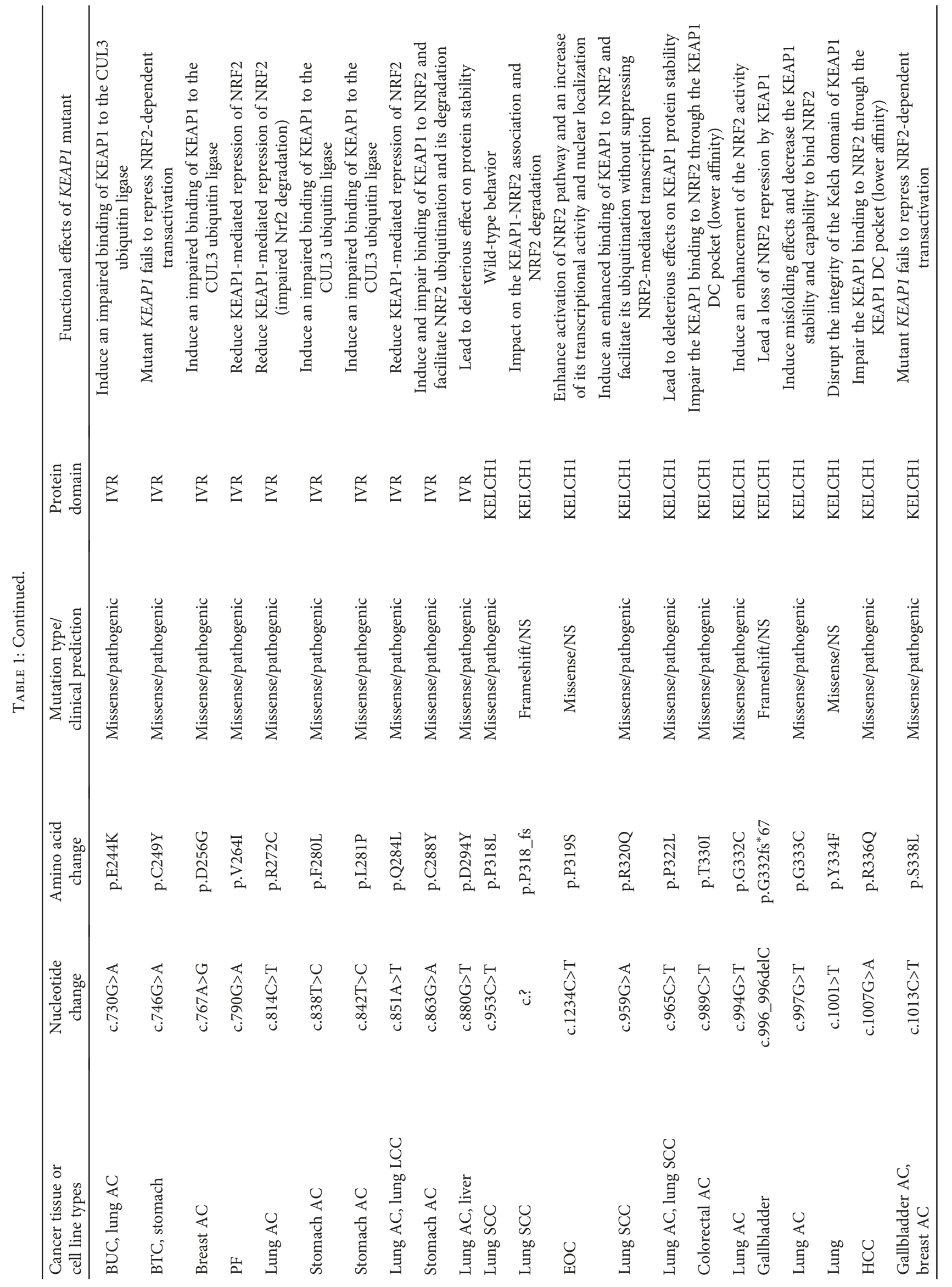




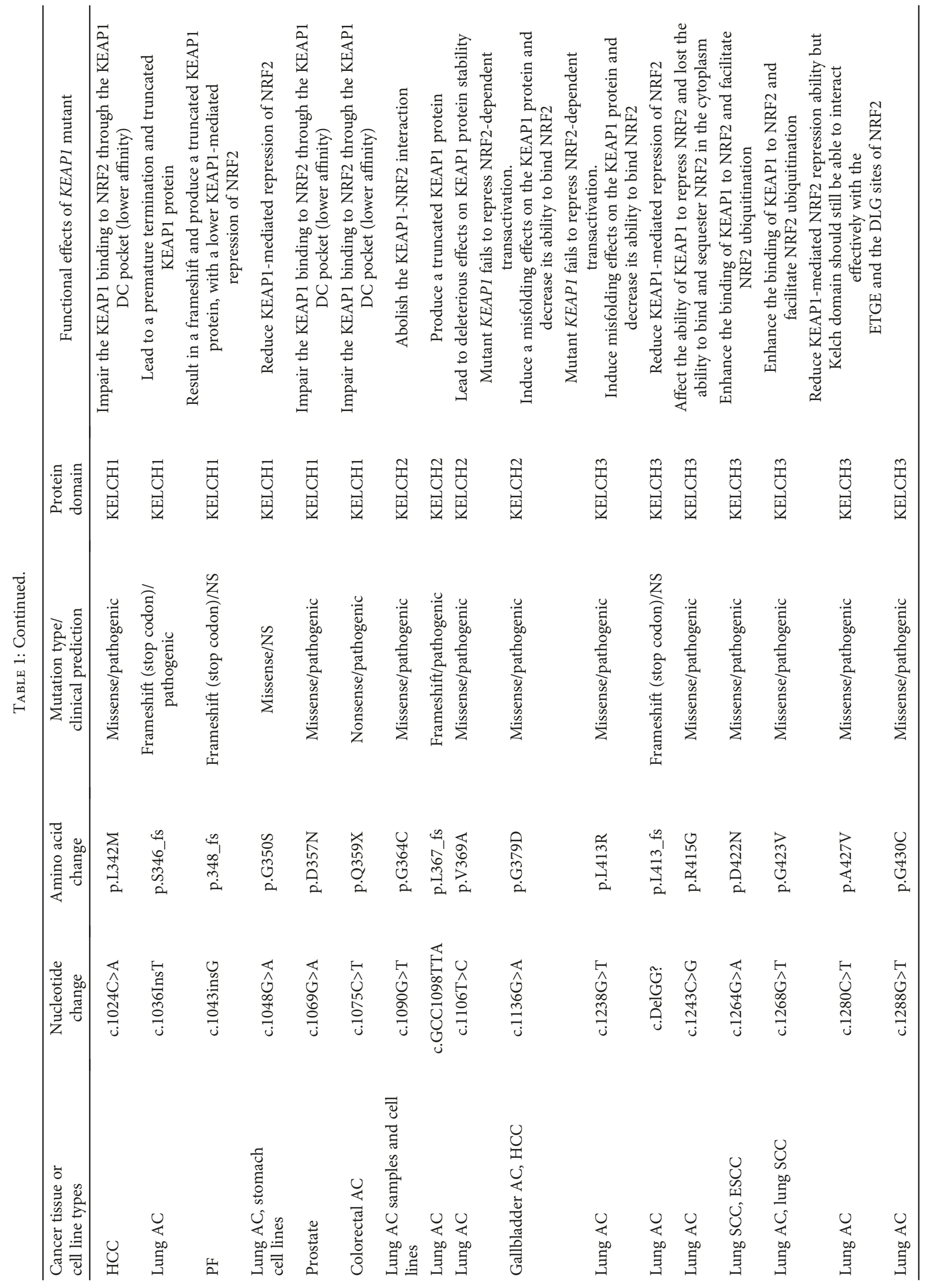




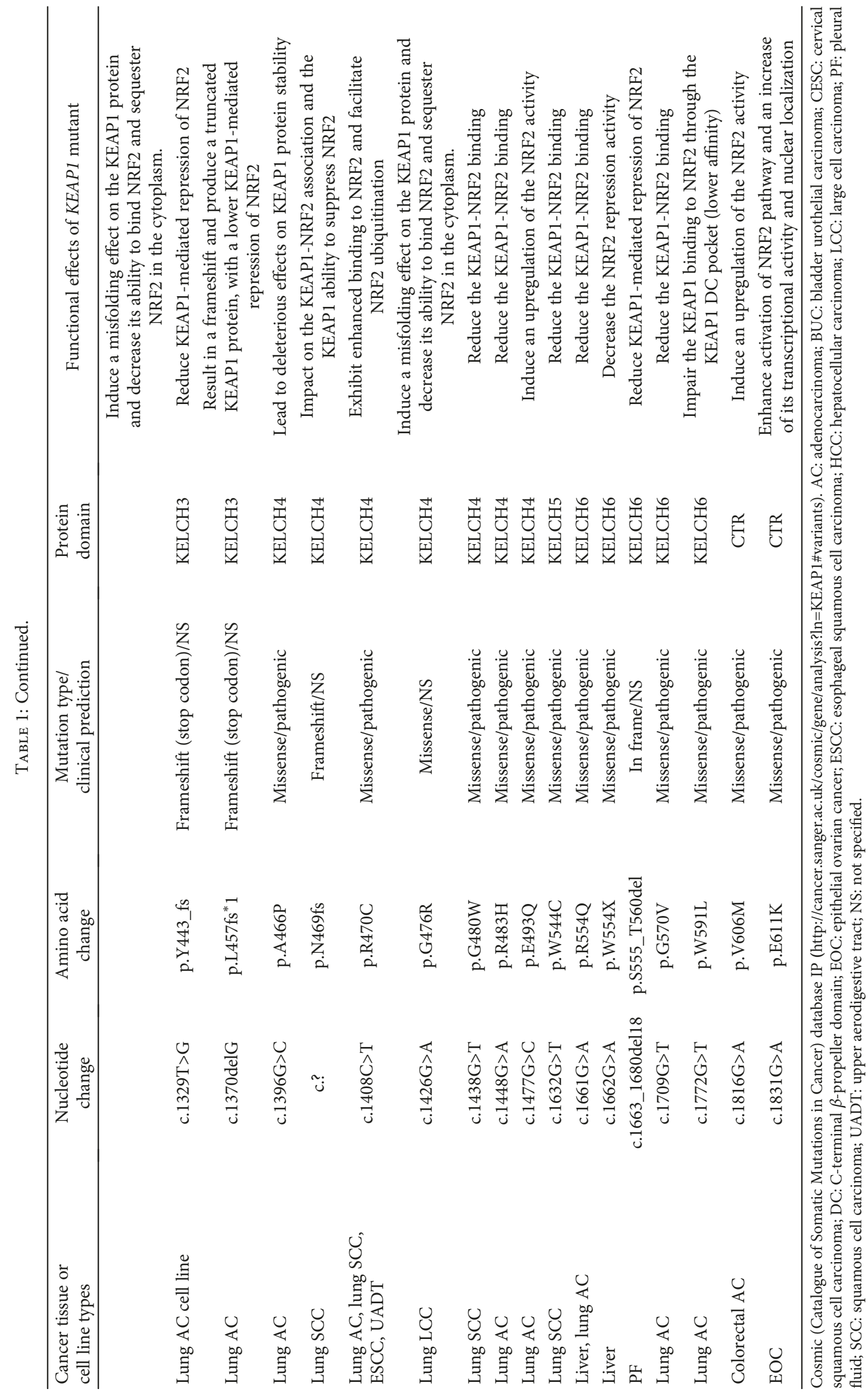




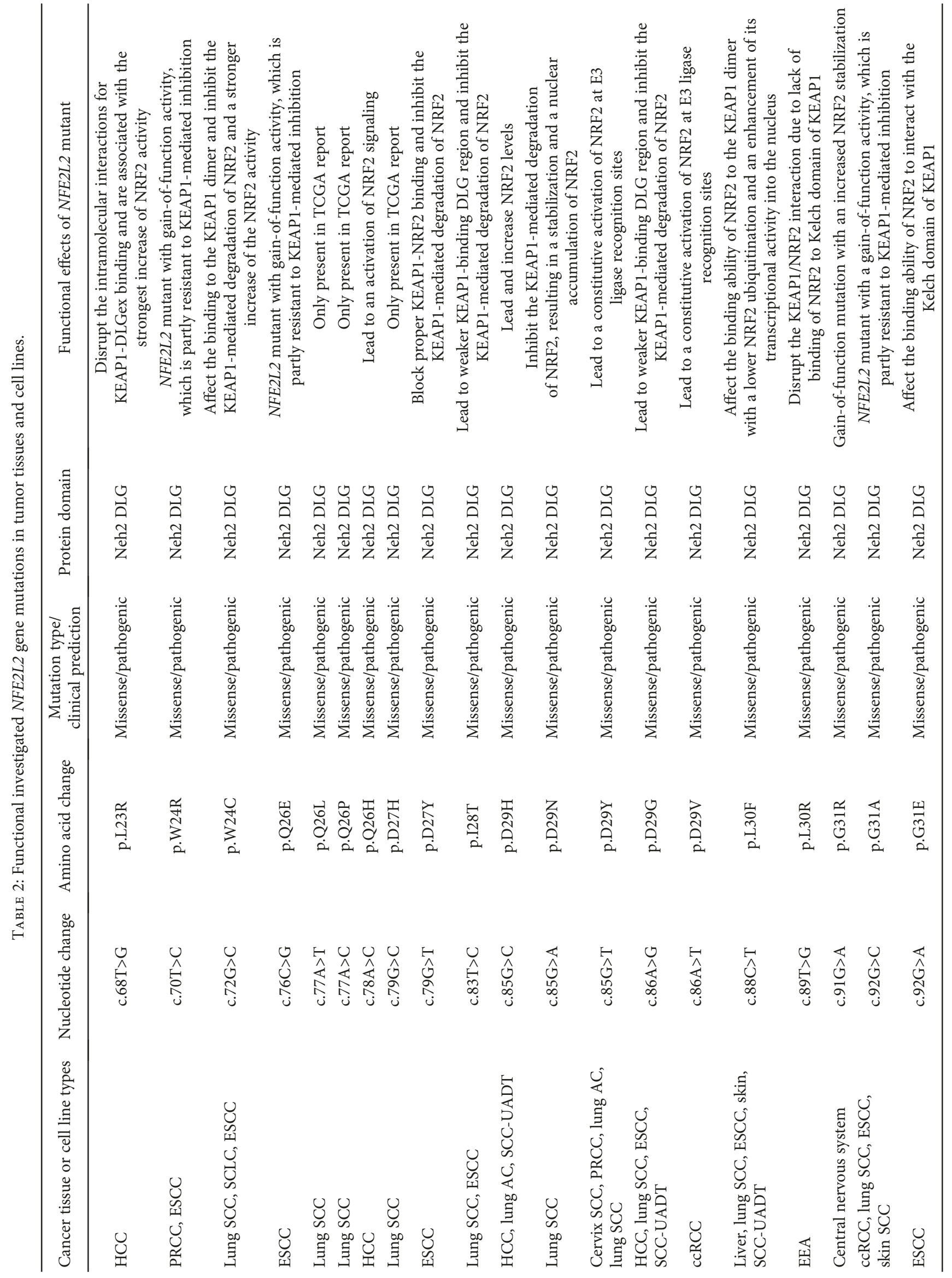




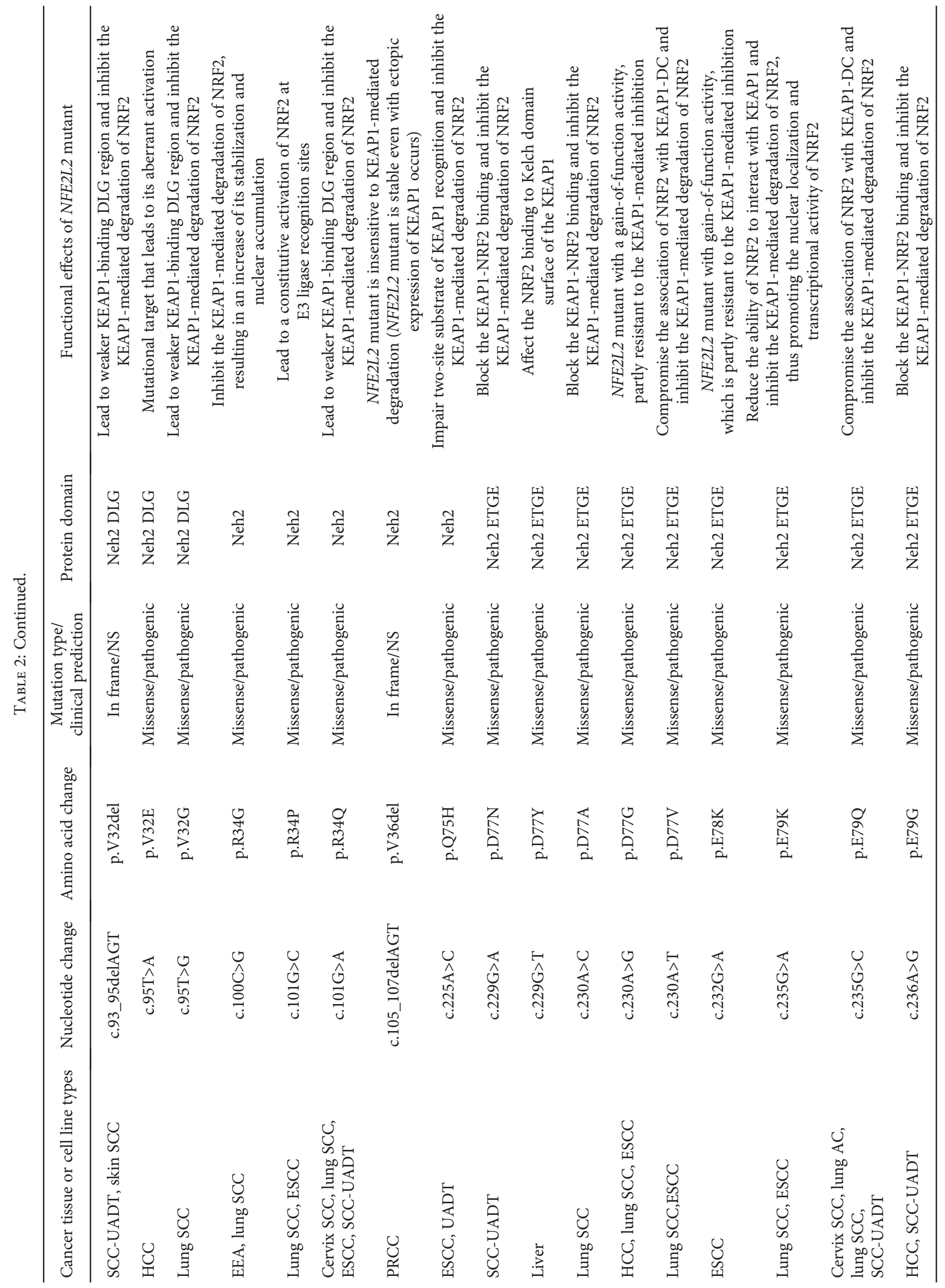




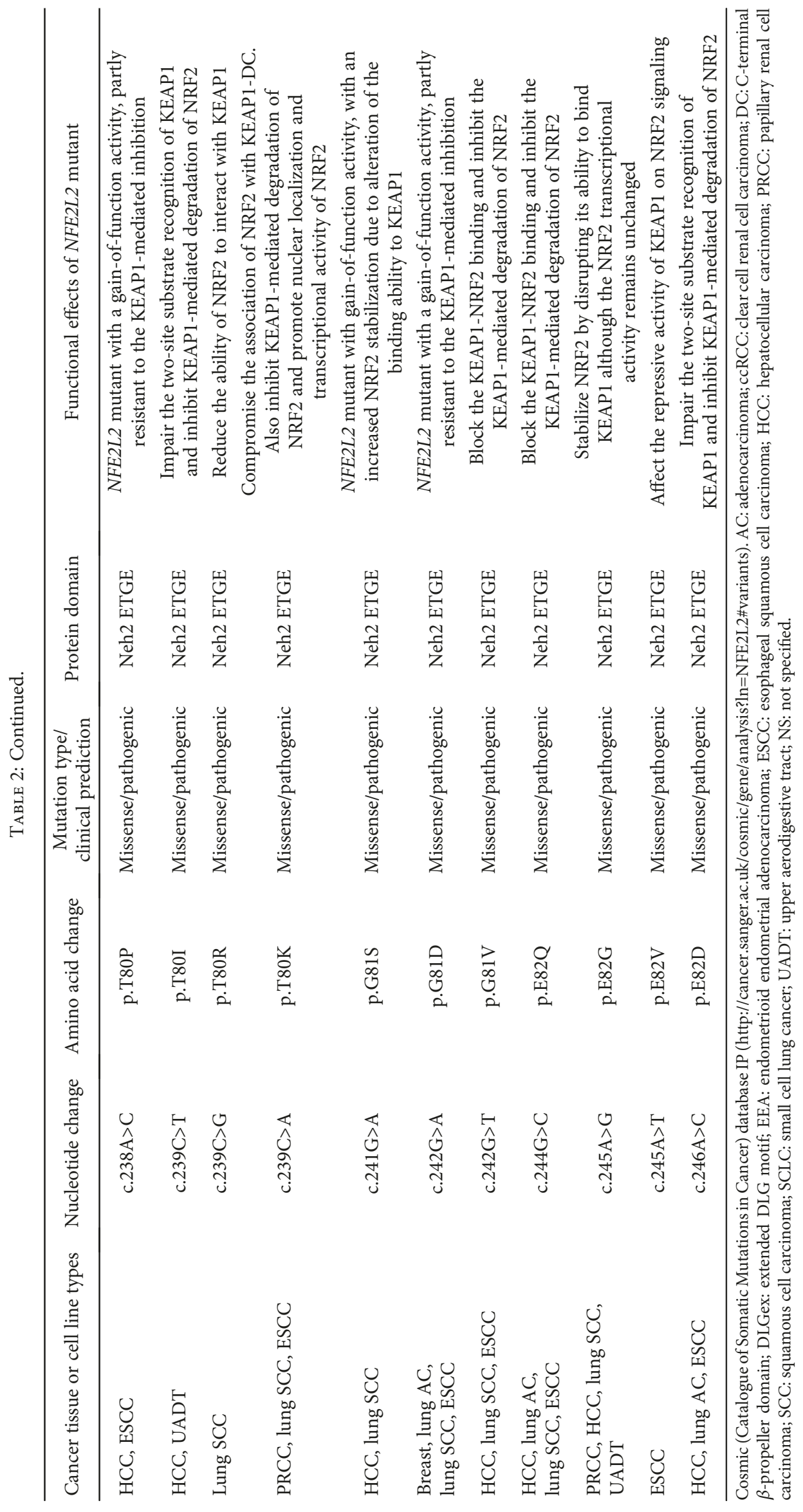




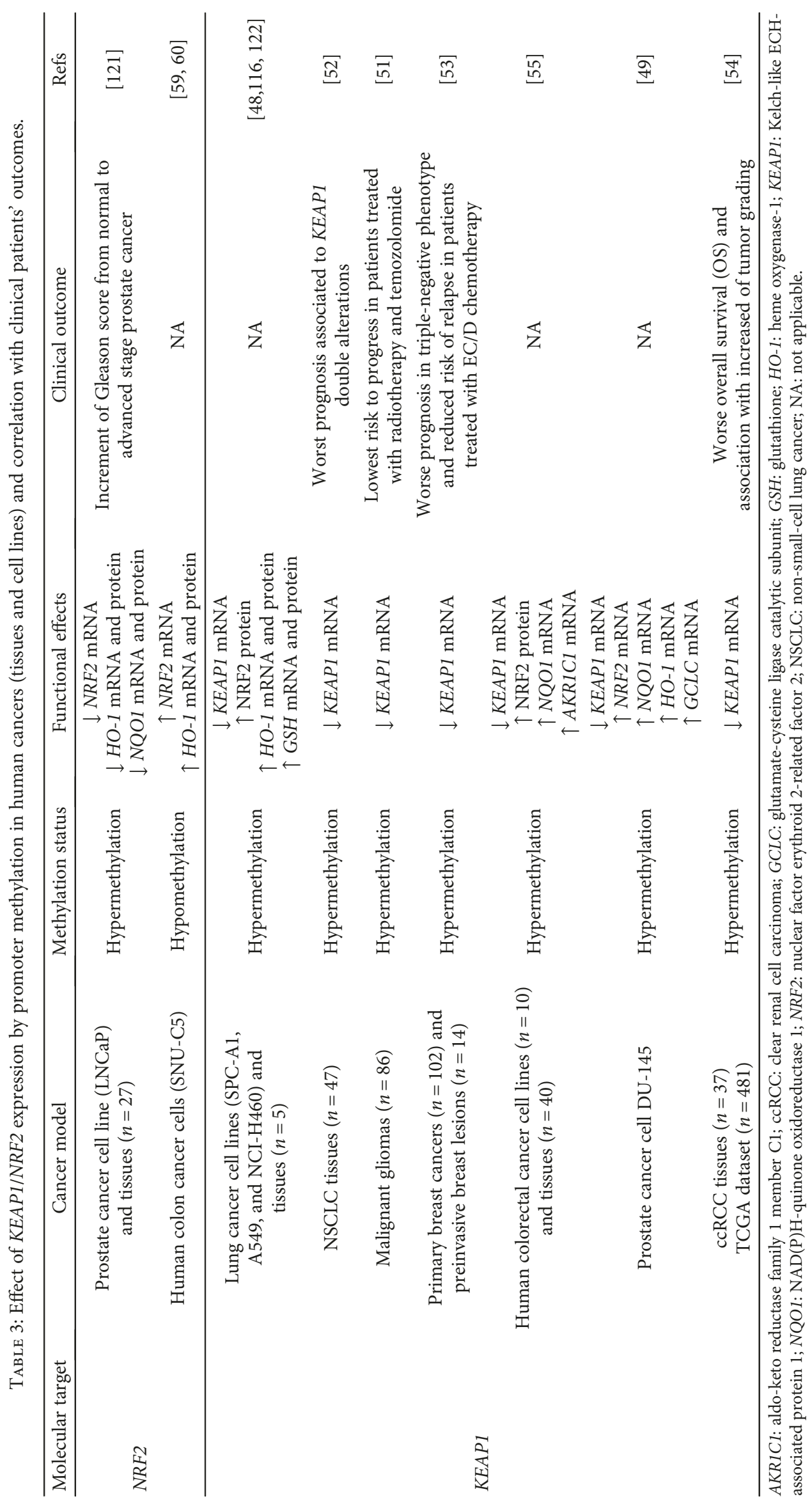


islands was demonstrated to induce an increase in transcript levels and a consequent overexpression of NRF2, GSS, and HO-1 [69].

Aberrant KEAP1 methylation was also reported in 53\% of colorectal cancer and head and neck cancer tissues (29.3\%) and was also linked to the worse prognosis of these tumors $[70,71]$. In pancreatic cancer cell lines, the suppression of KEAP1 protein was demonstrated to be correlated with UHRF1, a scaffold protein for DNA methyltransferase DNMT1 [72].

A possible role of epigenetic variations in the modulation of NRF2 expression is less investigated. $\mathrm{Li}$ and colleagues showed that a low methyltransferase EZH2 expression correlates both lung cancer cell lines and tissues with an elevated expression of NRF2, NQO1 (NAD(P)Hquinone oxidoreductase 1), and HO-1 (heme oxygenase 1). Since the EZH2 is involved in the establishment and/or maintenance of chromatin architecture and histone methylation, its downstream effect was attributed to a decrease in the trimethylation of lysine 27 on histone $\mathrm{H} 3(\mathrm{H} 3 \mathrm{~K} 27 \mathrm{Me} 3)$ in the NFE2L2 promoter region [73]. Recently, Kang and colleagues focused on the causative relationship between NRF2 expression and its epigenetic alterations, especially in the context of DNA methylation at cytosines and histone methylation status during 5-fluorouracil- (5-FU-) induced oxidative stress in colon cancer cells. They found that elevated reactive oxygen species (ROS) level induced by 5 -FU activates TET (ten-eleven translocation) DNA demethylases and produces a hypomethylation of the NFE2L2 promoter with consequent activation of NRF2 translation. This, in turn, upregulates the expression of the antioxidant enzymes and generates the resistance to $5-\mathrm{FU}$ in cancer cells $[48,49]$.

\section{MicroRNAs Directly Targeting the Keap1/ Nrf2 Pathway}

An intriguing epigenetic way of KEAP1/NRF2 pathway deregulation in tumor cells comes from miRNAs that act in cancer as oncogenes or tumor suppressors [74]. miRNAs are proximately 22 nucleotide single-stranded noncoding RNA molecules which regulate gene expression at posttranscriptional levels by binding to the $3^{\prime}$-untranslated regions (UTRs) of specific mRNAs. They generally affect the translation or stability of mRNA molecules through the interaction of specific mRNAs with complementary base pairing.

Two different blocks of miRNAs can be distinguished in the context of posttranscriptional regulation of the KEAP1/ NRF2 pathway (Table 4) [75]. The first group includes the miRNAs that directly target NFE2L2 and usually negatively regulate the KEAP1/NRF2 pathway by decreasing the NRF2 protein levels. The second one comprises those miRNAs that directly interact with KEAP1 and indirectly influence the NRF2 signaling. In addition, a lot of miRNAs are reported to indirectly modulate the ARE-mediated redox signaling through their interaction with additional factors located in the crossing points of the NRF2 network (Figure 3). All of these interactions are complex and still remain to be fully elucidated. So we focalized our attention on the main findings concerning NRF2 and KEAP1.

Several miRNAs predicted to target the KEAP1/NRF2 axis were identified by bioinformatic analysis of miRNA databases [76]. Some of these were experimentally proven to directly target and repress the NRF2 activity. The negative effects on the NRF2 expression by miR-28 were firstly documented in MCF-7 breast cancer cells [77]. A similar activity was described for miR-155 in 16-HBE human bronchiolar epithelial cells under arsenite treatment, together with an observed upregulation of glutathione (GSH), nitric oxide (NO), and superoxide dismutase (SOD) [78]. The increased expression of miR-155 correlates with radiation-induced severe fibrosis in a murine skin model [79], similar to miR140 , whose deficiency increased activation of TGF- $\beta 1$ signaling, inflammation, and myofibroblast differentiation in fibrotic lung tissue after radiation treatment [80, 81]. Among functionally validated miRNAs that regulate NRF2, miR-144 recently emerged as having a central role in the modulation of cellular stress response in blood malignancies and solid tumors. In K562 cell line and primary erythroid progenitor cells, it was seen that increased levels of miR-144 were associated with reduced NRF2 levels in HbSS reticulocytes. By contrast, inhibition of miR-144-3p in human leukemia HL60 cells reduced cell viability and prompted apoptosis by interfering with NRF2 activity. In hepatocellular carcinoma cell lines, miR-144 overexpression was reversely correlated with an enhancement of 5-fluorouracil- (5-FU-) induced cytotoxicity and apoptosis and with GSH biosynthesis in neuroblastoma SH-SY5Y cells via NRF2 [82-84].

A direct effect of miR-93 on nuclear accumulation of NRF2 was well described by Singh et al. using a rat model of breast carcinogenesis. A significant reduction in carcinogenesis-associated phenotypes such as mammosphere development, antiapoptosis, and DNA damage was observed [85].

In silico analysis and in vitro studies on different sets of tumor cell lines recently provided more additional insights into the role of KEAP1/NRF2 axis modulation by miRNAs. In SH-SY5Y neuroblastoma cells, the $3^{\prime}$-UTR of NFE2L2 is targeted by miR-153, miR27a, and miR-142-5p, with a consequent decrease in Gclc glutamate-cysteine ligase (GCLC) and glutathione-disulfide reductase (GSR) expression levels [86]. The functional impact of miR-153-3p/NRF2 interaction was firstly reported in breast cancer cell lines and recently highlighted by microarray studies in oral squamous cell carcinoma cell lines and tissues. Low expression levels of miR-153-3p significantly correlate with tumor cell migration and invasion $[87,88]$. In the same histological pattern of esophageal cell carcinoma, miR-340 was shown to directly modulate the NRF2 expression levels, thus interfering with the chemoresistance phenotype under cisplatin treatment [89]. Finally, miR-507, miR-634, miR-450a, and miR-129-5p appeared to negatively modulate the NRF2 activity by targeting both the NFE2L2 and ME1 transcripts, a well-known target of NRF2 [90]. In NSCLC A549 cells, this group of miRNAs exerts a synergic effect in increasing sensitivity of cell growth suppression under cisplatin treatment [91]. 


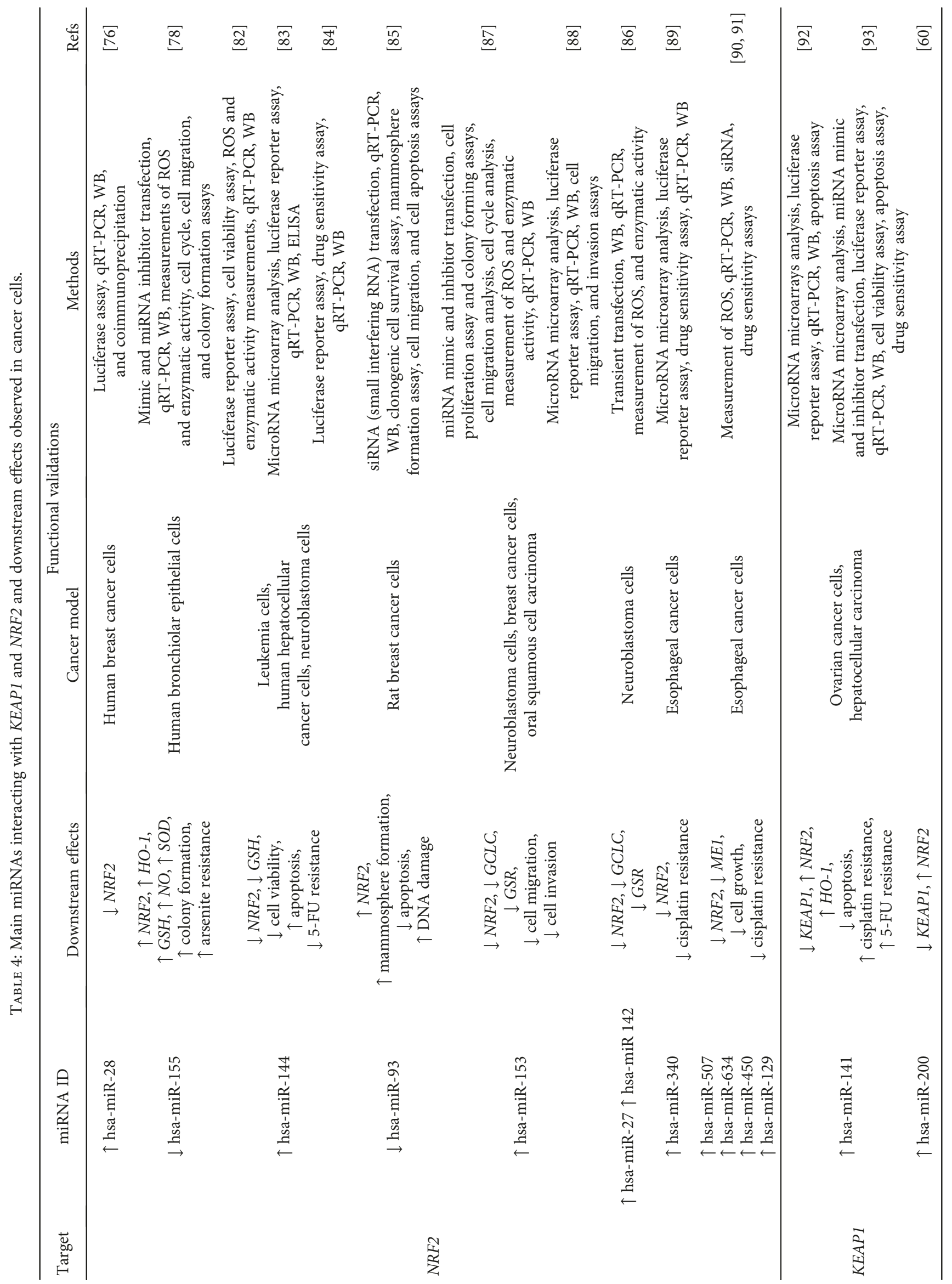




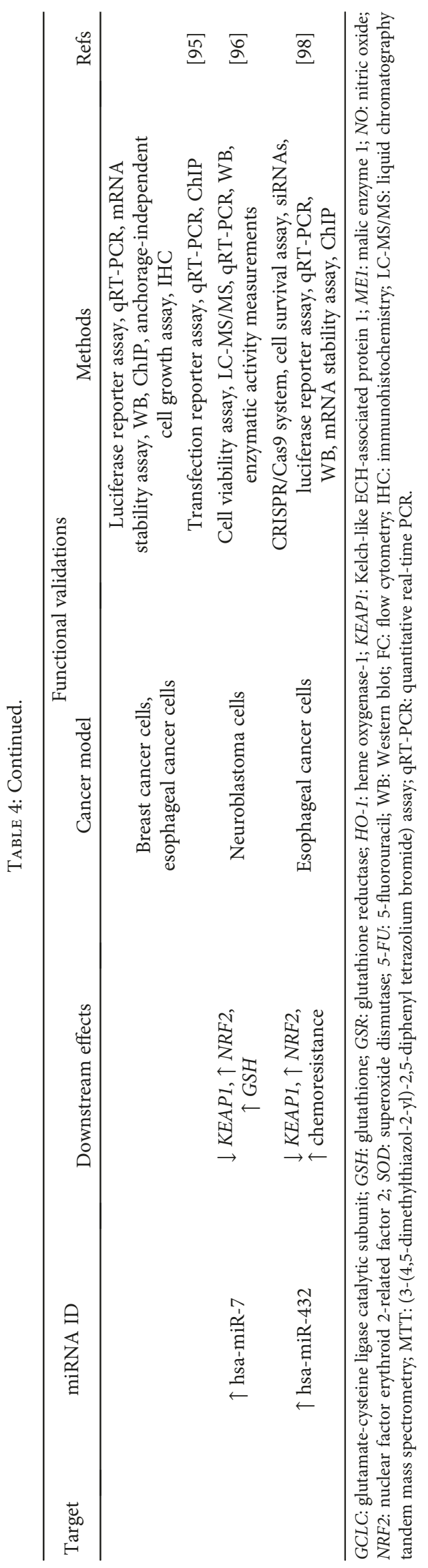




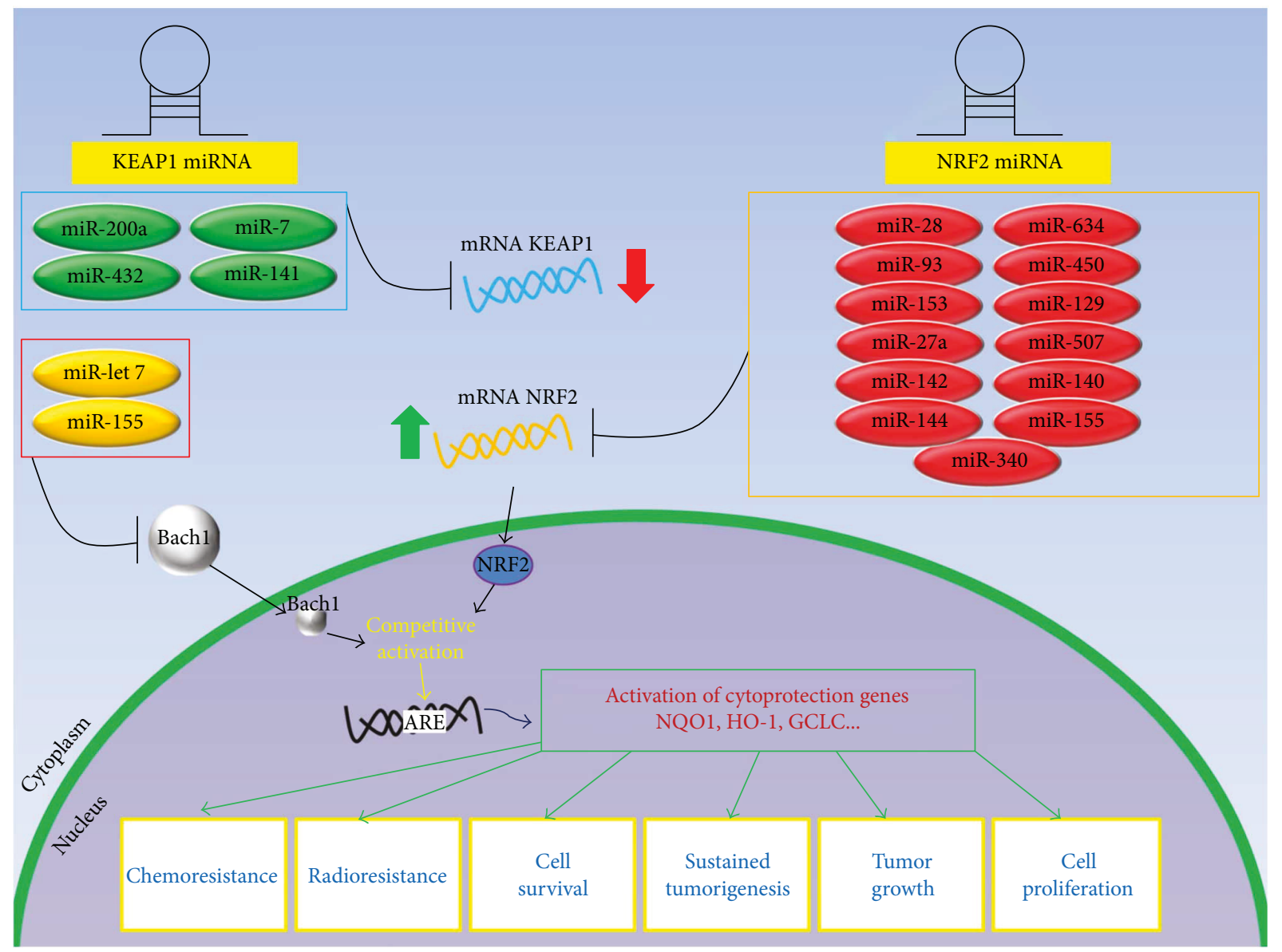

FIGURE 3: Left and right panels show how miRNA modifications may contribute to down and upregulate the KEAP1/NRF2 signaling in cancer. Representative scheme on the left side summarizes a group of miRNAs that directly target KEAP1 mRNA and indirectly impact on the transcriptional activity of the NRF2 into the nucleus. Other miRNAs modulate BACH1, a transcription factor that competes with NRF2 leading to the link at the antioxidant response element (ARE) of detoxifying genes. The schematic model on the right side depicts those miRNAs that directly target NFE2L2 and impact on the general mRNA and protein levels of NRF2 and, by consequence, on the activation of detoxification NRF2 target genes with a great impact on chemo- and radioresistance, survival, growth, and proliferation of tumor cells.

By looking at the KEAP1 regulation side, miR-141 was the first reported miRNA to target KEAP1 by binding to its $3^{\prime}$-UTR sequence site in ovarian carcinoma cell lines [92]. The upregulation of miR-141 expression decreases the 5-FU-mediated effects and apoptosis in hepatocellular carcinoma cell lines by inducing nuclear translocation of NRF2 and activation of HO-1 gene transcription [93]. A direct inhibition effect of miR-200a on KEAP1 was elucidated in human MDA-MB-231 and Hs578T breast and esophageal squamous cell carcinoma cells under methylseleninic acid (MSA) treatment. MSA acts as a chemopreventive agent that is able to induce miR-200a expression and inhibits KEAP1 through the Krüpple-like factor 4 (KLF4) [60, 94, 95]. Interesting results came from the investigation by Hartikainen et al. In their work, SNP rs1048290 has been found in LD with SNP rs9676881, which is located in a putative enhancer region, few bases downstream of the $3^{\prime}$-untranslated region $\left(3^{\prime}\right.$-UTR) of the KEAP1 gene, the specific target region of miR-200a [56]. More recently, a direct action of miR-7 on KEAP1 expression was described in the human neuroblastoma cells. By targeting the $3^{\prime}$-UTR of KEAP1 mRNA, miR-7 enhances the nuclear localization of NRF2 and induces an increased expression of HO- 1 and glutamate-cysteine ligase modifier subunit (GCLM). The control of cell survival under stress by miR-7 was amplified by the observed variation of intracellular hydroperoxide levels and increases in the reduced form of glutathione levels $[83,96]$. A similar effect was described for miR-196 in human hepatoma cells against hepatitis $\mathrm{C}$ virus infection [97]. The CRISPR/Cas9 system was used to prove the direct binding to the coding region of KEAP1 by miR432-3p in the esophageal squamous cell carcinoma (ESCC). In this tumor, miR-432-3p overexpression correlates with a downregulation of the KEAP1 expression, thus inducing a decrease in the sensitivity of tumor cells to cisplatin and other chemotherapy drugs [98].

A lot of miRNAs were also reported to regulate the KEAP1/NRF2 pathway independently from the KEAP1 or NRF2 activity. The let-7 family modulates the DICER expression and represents the first example of cancer regulation by miRNA in humans [99]. Not less importantly, let-7 
showed to inhibit the expression of several oncogenes involved in cellular proliferation, such as RAS (rat sarcoma), $M Y C$ (avian myelocytomatosis viral oncogene homolog), and HMGA2 (high-mobility group AT-hook 2) [100, 101]. The miRNAs let-7b and let-7c were firstly demonstrated to negatively modulate the expression of $\mathrm{BACH} 1$ in the liver, a transcription factor that works in association with the small MAF proteins in a dominant condition in respect of NRF2 [102, 103]. By consequence, the repression of $\mathrm{BACH} 1$ induces an upregulation of $\mathrm{HO}-1$ expression via NRF2 transcription [104].

\section{Long Noncoding RNAs Linked to the Keap1/ Nrf2 Pathway}

Long noncoding RNAs (lncRNAs) are non-protein-coding transcripts longer than 200 nucleotides which are expressed in a sense, antisense, or bidirectional manner. Different to protein-coding genes, they show a high density of DNA methylation around their transcription start sites, independent of their expression status [105]. A growing number of evidences elucidated the role of lncRNAs in the initiation, progression, and stem cell pluripotency of cancer cells [106]. Little is known about the role of lncRNAs in the modulation of the detoxification processes of cells.

The most recent findings are those related to smoke and cancer-associated lncRNA 1 (SCAL1) and lncRNA regulator of reprogramming (ROR). The SCAL1 is the first characterized long noncoding RNA activated by NRF2 and is considered one of the downstream mediators of NRF2-induced oxidative stress protection in airway epithelial cells. Under stress induced by cigarette smoke, the SCAL1 expression increases in lung cancer cell lines and appears to be directly correlated with NFE2L2 mutations [107]. In human bronchial epithelial cells, a knockdown gene approach revealed that NRF2 can regulate the expression level of SCAL1 by binding to the nuclear factor erythroid-derived 2 (NF-E2) motif located in the promoter region of its gene [108]. Conversely to SCAL1, Zhang and colleagues proved that in breast cells, NRF2 controls the ROR lncRNA expression by binding two different NRF2 response elements flanking the $R O R$ promoter region. NFE2L2 knockdown leads to the overexpression of IncRNA ROR in mammary embryonic stem cells [109].

\section{Concluding Remarks}

Significant advances have been made in these last years to understand the regulation mechanisms of the KEAP1/ NRF2 system. However, although KEAP1/NRF2 dysfunction is now well known to confer resistance to chemo- and radiotherapy, the KEAP1-NFE2L2 mutational status assessment is not used to make treatment decisions in lung cancer yet. Moreover, molecular profiling of these two proteins in pretreated and resistant tumor samples will help to elucidate if the loss of KEAP1 or the gain of NFE2L2 may be clinically relevant mechanisms of acquired and intrinsic resistance to therapies in lung cancer and other solid tumors or not. A general limitation to clarify these issues remains and consists of a lack of available rebiopsy tissue specimens.

From an epigenetic point of view, the effects produced by KEAP1 hypermethylation on the KEAP1/NRF2 signaling in cancer remain partially understood. Firstly, it is natural to wonder what the real role of $\mathrm{P} 1 \mathrm{CpG}$ island methylation is and if the methylation status of KEAP1 exclusively affects its expression or could additionally interfere with the ability to bind to NRF2 in promoting tumor progression and resistance to therapies. According to these observations, it would be of great interest to determine if in tumors with different origins there are similar or different methylation $\mathrm{CpG}$ density patterns at the $\mathrm{P} 1$ region and if demethylation of the KEAP1 promoter in neoplastic tissues could really suppress tumor progression and enhance resistance to therapies.

Given that posttranscriptional modifications play important roles in regulating the stability and translation of mRNAs, more studies on the regulation of the KEAP1/ NRF2 pathway by miRNAs will corroborate their key roles in clinical practice. However, this approach will require a greater knowledge of how drug treatment influences miRNA expression and how miRNA expression could influence the multifaced KEAP1/NRF2 network.

\section{Conflicts of Interest}

The authors declare that they have no competing financial interests.

\section{Acknowledgments}

This work was supported by the Italian Ministry of Health (Ricerca Corrente, RC1703LO41 to L.A. Muscarella) by " $5 \times 1000$ " voluntary contributions to Casa Sollievo della Sofferenza and AIRC/MFAG Grant 12983 (to L.A. Muscarella).

\section{References}

[1] K. Taguchi and M. Yamamoto, "The KEAP1-NRF2 system in cancer," Frontiers in Oncology, vol. 7, p. 85, 2017.

[2] K. Itoh, P. Ye, T. Matsumiya, K. Tanji, and T. Ozaki, "Emerging functional cross-talk between the Keap1-Nrf2 system and mitochondria," Journal of Clinical Biochemistry and Nutrition, vol. 56, no. 2, pp. 91-97, 2015.

[3] K. E. Hawkins, S. Joy, J. M. K. M. Delhove et al., "NRF2 orchestrates the metabolic shift during induced pluripotent stem cell reprogramming," Cell Reports, vol. 14, no. 8, pp. 1883-1891, 2016.

[4] A. Sparaneo, F. P. Fabrizio, and L. A. Muscarella, "Nrf2 and notch signaling in lung cancer: near the crossroad," Oxidative Medicine and Cellular Longevity, vol. 2016, Article ID 7316492, 17 pages, 2016.

[5] M. Mcmahon, K. Itoh, M. Yamamoto, and J. D. Hayes, "Keap1-dependent proteasomal degradation of transcription factor Nrf2 contributes to the negative regulation of antioxidant response element-driven gene expression," The Journal of Biological Chemistry, vol. 278, no. 24, pp. 21592-21600, 2003. 
[6] H. K. Bryan, A. Olayanju, C. E. Goldring, and B. K. Park, "The Nrf2 cell defence pathway: Keap1-dependent and -independent mechanisms of regulation," Biochemical Pharmacology, vol. 85, no. 6, pp. 705-717, 2013.

[7] K. Taguchi, H. Motohashi, and M. Yamamoto, "Molecular mechanisms of the Keap1-Nrf2 pathway in stress response and cancer evolution," Genes to Cells, vol. 16, no. 2, pp. 123-140, 2011.

[8] L. M. Solis, C. Behrens, W. Dong et al., "Nrf2 and Keap1 abnormalities in non-small cell lung carcinoma and association with clinicopathologic features," Clinical Cancer Research, vol. 16, no. 14, pp. 3743-3753, 2010.

[9] M. H. Chien, W. J. Lee, F. K. Hsieh et al., "Keap1-Nrf2 interaction suppresses cell motility in lung adenocarcinomas by targeting the S100P protein," Clinical Cancer Research, vol. 21, no. 20, pp. 4719-4732, 2015.

[10] A. Singh, V. Misra, R. K. Thimmulappa et al., "Dysfunctional KEAP1-NRF2 interaction in non-small-cell lung cancer," PLoS Medicine, vol. 3, no. 10, article e420, 2006.

[11] J. D. Hayes and M. Mcmahon, "NRF2 and KEAP1 mutations: permanent activation of an adaptive response in cancer," Trends in Biochemical Sciences, vol. 34, no. 4, pp. 176-188, 2009.

[12] D. Guo, B. Wu, J. Yan, X. Li, H. Sun, and D. Zhou, “A possible gene silencing mechanism: hypermethylation of the Keap1 promoter abrogates binding of the transcription factor Sp1 in lung cancer cells," Biochemical and Biophysical Research Communications, vol. 428, no. 1, pp. 80-85, 2012.

[13] Y. Mitsuishi, H. Motohashi, and M. Yamamoto, "The Keap1Nrf2 system in cancers: stress response and anabolic metabolism," Frontiers in Oncology, vol. 2, p. 200, 2012.

[14] B. Padmanabhan, K. I. Tong, T. Ohta et al., "Structural basis for defects of Keap1 activity provoked by its point mutations in lung cancer," Molecular Cell, vol. 21, no. 5, pp. 689-700, 2006.

[15] H. Tian, B. Zhang, J. Di et al., "Keap1: one stone kills three birds Nrf2, IKK $\beta$ and Bcl-2/Bcl-xL," Cancer Letters, vol. 325, no. 1, pp. 26-34, 2012.

[16] C. B. Lebovitz, A. G. Robertson, R. Goya et al., "Cross-cancer profiling of molecular alterations within the human autophagy interaction network," Autophagy, vol. 11, no. 9, pp. 1668-1687, 2015.

[17] R. J. G. Cardnell, C. Behrens, L. Diao et al., “An integrated molecular analysis of lung adenocarcinomas identifies potential therapeutic targets among TTF1-negative tumors, including DNA repair proteins and Nrf2," Clinical Cancer Research, vol. 21, no. 15, pp. 3480-3491, 2015.

[18] Q. K. Li, A. Singh, S. Biswal, F. Askin, and E. Gabrielson, "KEAP1 gene mutations and NRF2 activation are common in pulmonary papillary adenocarcinoma," Journal of Human Genetics, vol. 56, no. 3, pp. 230-234, 2011.

[19] N. J. Yoo, H. R. Kim, Y. R. Kim, C. H. An, and S. H. Lee, "Somatic mutations of the KEAP1 gene in common solid cancers," Histopathology, vol. 60, no. 6, pp. 943-952, 2012.

[20] T. Shibata, A. Kokubu, M. Gotoh et al., "Genetic alteration of Keap1 confers constitutive Nrf2 activation and resistance to chemotherapy in gallbladder cancer," Gastroenterology, vol. 135, no. 4, pp. 1358-1368.e4, 2008.

[21] P. A. Konstantinopoulos, D. Spentzos, E. Fountzilas et al., "Keap1 mutations and Nrf2 pathway activation in epithelial ovarian cancer," Cancer Research, vol. 71, no. 15, pp. 5081-5089, 2011.

[22] M. Kanamori, T. Higa, Y. Sonoda et al., "Activation of the NRF2 pathway and its impact on the prognosis of anaplastic glioma patients," Neuro-Oncology, vol. 17, no. 4, pp. 555-565, 2015.

[23] P. Nioi and T. Nguyen, "A mutation of Keap1 found in breast cancer impairs its ability to repress Nrf2 activity," Biochemical and Biophysical Research Communications, vol. 362, no. 4, pp. 816-821, 2007.

[24] T. Sjoblom, S. Jones, L. D. Wood et al., "The consensus coding sequences of human breast and colorectal cancers," Science, vol. 314, no. 5797, pp. 268-274, 2006.

[25] V. D. Martinez, E. A. Vucic, K. L. Thu, L. A. Pikor, S. Lam, and W. L. Lam, "Disruption of KEAP1/CUL3/RBX1 E3ubiquitin ligase complex components by multiple genetic mechanisms: association with poor prognosis in head and neck cancer," Head \& Neck, vol. 37, no. 5, pp. 727-734, 2015.

[26] Y. Sato, T. Yoshizato, Y. Shiraishi et al., "Integrated molecular analysis of clear-cell renal cell carcinoma," Nature Genetics, vol. 45, no. 8, pp. 860-867, 2013.

[27] M. Eichenmüller, F. Trippel, M. Kreuder et al., "The genomic landscape of hepatoblastoma and their progenies with HCClike features," Journal of Hepatology, vol. 61, no. 6, pp. 1312 1320, 2014.

[28] K. Schulze, S. Imbeaud, E. Letouzé et al., "Exome sequencing of hepatocellular carcinomas identifies new mutational signatures and potential therapeutic targets," Nature Genetics, vol. 47, no. 5, pp. 505-511, 2015.

[29] J. Zucman-Rossi, A. Villanueva, J. C. Nault, and J. M. Llovet, "Genetic landscape and biomarkers of hepatocellular carcinoma," Gastroenterology, vol. 149, no. 5, pp. 1226-1239.e4, 2015.

[30] L. Fernandez-Cuesta, M. Peifer, X. Lu et al., "Abstract 1531: crossentity mutation analysis of lung neuroendocrine tumors sheds light into their molecular origin and identifies new therapeutic targets," Cancer Research, vol. 74, no. 19, p. $1531,2014$.

[31] J. Derks, "Genetic subtypes of large cell neuroendocrine carcinoma (LCNEC) to predict response to chemotherapy," Journal of Clinical Oncology, vol. 35, Supplement 15, pp. 9061-9061, 2017.

[32] J. George, J. S. Lim, S. J. Jang et al., "Comprehensive genomic profiles of small cell lung cancer," Nature, vol. 524, no. 7563, pp. 47-53, 2015.

[33] D. W. Ball, "Achaete-scute homolog-1 and notch in lung neuroendocrine development and cancer," Cancer Letters, vol. 204, no. 2, pp. 159-169, 2004.

[34] The Cancer Genome Atlas Research Network, "Comprehensive genomic characterization of squamous cell lung cancers," Nature, vol. 489, no. 7417, pp. 519-525, 2012.

[35] H. Sasaki, "Increased NRF2 gene (NFE2L2) copy number correlates with mutations in lung squamous cell carcinomas," Molecular Medicine Reports, vol. 6, no. 2, pp. 391-394, 2012.

[36] Y. R. Kim, J. E. Oh, M. S. Kim et al., "Oncogenic NRF2 mutations in squamous cell carcinomas of oesophagus and skin," The Journal of Pathology, vol. 220, no. 4, pp. 446-451, 2010.

[37] L. D. Goldstein, J. Lee, F. Gnad et al., "Recurrent loss of NFE2L2 exon 2 is a mechanism for Nrf2 pathway activation in human cancers," Cell Reports, vol. 16, no. 10, pp. 2605$2617,2016$. 
[38] D. D. Zhang, S. C. Lo, Z. Sun, G. M. Habib, M. W. Lieberman, and M. Hannink, "Ubiquitination of Keap1, a BTB-Kelch substrate adaptor protein for Cul3, targets Keap1 for degradation by a proteasome-independent pathway," The Journal of Biological Chemistry, vol. 280, no. 34, pp. 30091-30099, 2005.

[39] M. Kovac, C. Navas, S. Horswell et al., "Recurrent chromosomal gains and heterogeneous driver mutations characterise papillary renal cancer evolution," Nature Communications, vol. 6, no. 1, p. 6336, 2015.

[40] A. Ooi, J.-C. Wong, D. Petillo et al., “An antioxidant response phenotype shared between hereditary and sporadic type 2 papillary renal cell carcinoma," Cancer Cell, vol. 20, no. 4, pp. 511-523, 2011.

[41] A. Ooi, K. Dykema, A. Ansari et al., "CUL3 and NRF2 mutations confer an NRF2 activation phenotype in a sporadic form of papillary renal cell carcinoma," Cancer Research, vol. 73, no. 7, pp. 2044-2051, 2013.

[42] S. Menegon, A. Columbano, and S. Giordano, "The dual roles of NRF2 in cancer," Trends in Molecular Medicine, vol. 22, no. 7, pp. 578-593, 2016.

[43] X. J. Wang, J. D. Hayes, and C. R. Wolf, "Generation of a stable antioxidant response element-driven reporter gene cell line and its use to show redox-dependent activation of nrf2 by cancer chemotherapeutic agents," Cancer Research, vol. 66, no. 22, pp. 10983-10994, 2006.

[44] H. Yang, W. Wang, Y. Zhang et al., "The role of NF-E2related factor 2 in predicting chemoresistance and prognosis in advanced non-small-cell lung cancer," Clinical Lung Cancer, vol. 12, no. 3, pp. 166-171, 2011.

[45] D. W. Cescon, D. She, S. Sakashita et al., "NRF2 pathway activation and adjuvant chemotherapy benefit in lung squamous cell carcinoma," Clinical Cancer Research, vol. 21, no. 11, pp. 2499-2505, 2015.

[46] J. Isohookana, K. M. Haapasaari, Y. Soini, and P. Karihtala, "Keap1 expression has independent prognostic value in pancreatic adenocarcinomas," Diagnostic Pathology, vol. 10, no. 1, p. 28, 2015.

[47] A. Lister, T. Nedjadi, N. R. Kitteringham et al., "Nrf2 is overexpressed in pancreatic cancer: implications for cell proliferation and therapy," Molecular Cancer, vol. 10, no. 1, p. 37, 2011

[48] K. A. Kang, M. J. Piao, K. C. Kim et al., "Epigenetic modification of Nrf2 in 5-fluorouracil-resistant colon cancer cells: involvement of TET-dependent DNA demethylation," Cell Death \& Disease, vol. 5, no. 4, article e1183, 2014.

[49] K. A. Kang, M. J. Piao, Y. S. Ryu et al., "Interaction of DNA demethylase and histone methyltransferase upregulates Nrf2 in 5-fluorouracil-resistant colon cancer cells," Oncotarget, vol. 7, no. 26, pp. 40594-40620, 2016.

[50] Y. Jeong, N. T. Hoang, A. Lovejoy et al., "Role of KEAP1/ NRF2 and TP53 mutations in lung squamous cell carcinoma development and radiation resistance," Cancer Discovery, vol. 7, no. 1, pp. 86-101, 2017.

[51] Q. Wang, J. Ma, Y. Lu et al., "CDK20 interacts with KEAP1 to activate NRF2 and promotes radiochemoresistance in lung cancer cells," Oncogene, vol. 36, no. 37, pp. 5321-5330, 2014.

[52] G. M. Denicola, F. A. Karreth, T. J. Humpton et al., "Oncogene-induced Nrf2 transcription promotes ROS detoxification and tumorigenesis," Nature, vol. 475, no. 7354, pp. 106-109, 2011.
[53] T. Yamadori, Y. Ishii, S. Homma et al., "Molecular mechanisms for the regulation of Nrf2-mediated cell proliferation in non-small-cell lung cancers," Oncogene, vol. 31, no. 45, pp. 4768-4777, 2012.

[54] Y. Huang, W. Li, Z.-Y. Su, and A.-N. T. Kong, "The complexity of the Nrf2 pathway: beyond the antioxidant response," The Journal of Nutritional Biochemistry, vol. 26, no. 12, pp. 1401-1413, 2015.

[55] E. B. Krall, B. Wang, D. M. Munoz et al., "KEAP1 loss modulates sensitivity to kinase targeted therapy in lung cancer," eLife, vol. 6, article e18970, 2017.

[56] J. M. Hartikainen, M. Tengström, R. Winqvist et al., "KEAP1 genetic polymorphisms associate with breast cancer risk and survival outcomes," Clinical Cancer Research, vol. 21, no. 7, pp. 1591-1601, 2015.

[57] L. A. Muscarella and V. M. Fazio, "Keap1/Nrf2 impairing revised: are we missing the single nucleotide polymorphisms?," Journal of Thoracic Disease, vol. 8, no. 12, pp. E1752-E1754, 2016.

[58] M. S. Nicoloso, H. Sun, R. Spizzo et al., "Single-nucleotide polymorphisms inside microRNA target sites influence tumor susceptibility," Cancer Research, vol. 70, no. 7, pp. 2789-2798, 2010.

[59] N. Zhao, J. G. Han, C. R. Shyu, and D. Korkin, "Determining effects of non-synonymous SNPs on protein-protein interactions using supervised and semi-supervised learning," PLoS Computational Biology, vol. 10, no. 5, article e1003592, 2014.

[60] G. Eades, M. Yang, Y. Yao, Y. Zhang, and Q. Zhou, "miR200a regulates Nrf2 activation by targeting Keap1 mRNA in breast cancer cells," The Journal of Biological Chemistry, vol. 286, no. 47, pp. 40725-40733, 2011.

[61] I. M. Copple, "The Keap1-Nrf2 cell defense pathway - a promising therapeutic target?," Advances in Pharmacology, vol. 63, pp. 43-79, 2016.

[62] R. Wang, J. An, F. Ji, H. Jiao, H. Sun, and D. Zhou, "Hypermethylation of the Keap1 gene in human lung cancer cell lines and lung cancer tissues," Biochemical and Biophysical Research Communications, vol. 373, no. 1, pp. 151-154, 2008.

[63] P. Zhang, A. Singh, S. Yegnasubramanian et al., "Loss of Kelch-like ECH-associated protein 1 function in prostate cancer cells causes chemoresistance and radioresistance and promotes tumor growth," Molecular Cancer Therapeutics, vol. 9, no. 2, pp. 336-346, 2010.

[64] T. O. Khor, F. Fuentes, L. Shu et al., "Epigenetic DNA methylation of antioxidative stress regulator NRF2 in human prostate cancer," Cancer Prevention Research, vol. 7, no. 12, pp. 1186-1197, 2014.

[65] L. A. Muscarella, R. Barbano, V. D’Angelo et al., "Regulation of KEAP1 expression by promoter methylation in malignant gliomas and association with patient's outcome," Epigenetics, vol. 6, no. 3, pp. 317-325, 2011.

[66] L. A. Muscarella, P. Parrella, V. D’Alessandro et al., "Frequent epigenetics inactivation of KEAP1 gene in non-small cell lung cancer," Epigenetics, vol. 6, no. 6, pp. 710-719, 2011.

[67] R. Barbano, L. A. Muscarella, B. Pasculli et al., "Aberrant Keap1 methylation in breast cancer and association with clinicopathological features," Epigenetics, vol. 8, no. 1, pp. 105-112, 2013.

[68] F. P. Fabrizio, M. Costantini, M. Copetti et al., "Keap1/Nrf2 pathway in kidney cancer: frequent methylation of KEAP1 
gene promoter in clear renal cell carcinoma," Oncotarget, vol. 8, no. 7, pp. 11187-11198, 2017.

[69] X. Liu, C. Sun, B. Liu et al., "Genistein mediates the selective radiosensitizing effect in NSCLC A549 cells via inhibiting methylation of the keap1 gene promoter region," Oncotarget, vol. 7, no. 19, pp. 27267-27279, 2016.

[70] N. Hanada, T. Takahata, Q. Zhou et al., "Methylation of the KEAP1 gene promoter region in human colorectal cancer," BMC Cancer, vol. 12, no. 1, p. 66, 2012.

[71] A. R. Jazirehi, P. B. Wenn, and D. Arle, "Is there a decrease in Keap1 RNA expression in colorectal cancer cells, and is this decrease in expression due to hypermethylation?," Epigenomics, vol. 4, no. 3, pp. 253-254, 2012.

[72] W. Abu-Alainin, T. Gana, T. Liloglou et al., "UHRF1 regulation of the Keap1-Nrf2 pathway in pancreatic cancer contributes to oncogenesis," The Journal of Pathology, vol. 238, no. 3, pp. 423-433, 2016.

[73] Z. Li, L. Xu, N. Tang et al., "The polycomb group protein EZH2 inhibits lung cancer cell growth by repressing the transcription factor Nrf2," FEBS Letters, vol. 588, no. 17, pp. 3000-3007, 2014.

[74] I. Berindan-Neagoe, C. Monroig Pdel, B. Pasculli, and G. A. Calin, "MicroRNAome genome: a treasure for cancer diagnosis and therapy," CA: A Cancer Journal for Clinicians, vol. 64, no. 5, pp. 311-336, 2014.

[75] D. Ayers, B. Baron, and T. Hunter, "miRNA influences in NRF2 pathway interactions within cancer models," Journal of Nucleic Acids, vol. 2015, Article ID 143636, 6 pages, 2015.

[76] D. Papp, K. Lenti, D. Módos et al., “The NRF2-related interactome and regulome contain multifunctional proteins and fine-tuned autoregulatory loops," FEBS Letters, vol. 586, no. 13, pp. 1795-1802, 2012.

[77] M. Yang, Y. Yao, G. Eades, Y. Zhang, and Q. Zhou, "MiR-28 regulates Nrf2 expression through a Keap1-independent mechanism," Breast Cancer Research and Treatment, vol. 129, no. 3, pp. 983-991, 2011.

[78] C. Chen, X. Jiang, S. Gu, and Z. Zhang, "MicroRNA-155 regulates arsenite-induced malignant transformation by targeting Nrf2-mediated oxidative damage in human bronchial epithelial cells," Toxicology Letters, vol. 278, pp. 38-47, 2017.

[79] N. Pottier, T. Maurin, B. Chevalier et al., "Identification of keratinocyte growth factor as a target of microRNA-155 in lung fibroblasts: implication in epithelial-mesenchymal interactions," PLoS One, vol. 4, no. 8, article e6718, 2009.

[80] N. Duru, R. Gernapudi, Y. Zhang et al., "NRF2/miR-140 signaling confers radioprotection to human lung fibroblasts," Cancer Letters, vol. 369, no. 1, pp. 184-191, 2015.

[81] N. Duru, Y. Zhang, R. Gernapudi et al., "Loss of miR-140 is a key risk factor for radiation-induced lung fibrosis through reprogramming fibroblasts and macrophages," Scientific Reports, vol. 6, no. 1, article 39572, 2017.

[82] C. Zhou, L. Zhao, J. Zheng et al., "MicroRNA-144 modulates oxidative stress tolerance in SH-SY5Y cells by regulating nuclear factor erythroid 2-related factor 2-glutathione axis," Neuroscience Letters, vol. 655, pp. 21-27, 2016.

[83] X. Sun, D. Liu, Y. Xue, and X. Hu, "Enforced miR-144-3p expression as a non-invasive biomarker for the acute myeloid leukemia patients mainly by targeting NRF2," Clinical Laboratory, vol. 63, no. 4, pp. 679-687, 2017.

[84] S. Zhou, W. Ye, Y. Zhang et al., "miR-144 reverses chemoresistance of hepatocellular carcinoma cell lines by targeting
Nrf2-dependent antioxidant pathway," American Journal of Translational Research, vol. 8, no. 7, pp. 2992-3002, 2010.

[85] B. Singh, A. M. Ronghe, A. Chatterjee, N. K. Bhat, and H. K. Bhat, "MicroRNA-93 regulates NRF2 expression and is associated with breast carcinogenesis," Carcinogenesis, vol. 34, no. 5, pp. 1165-1172, 2013.

[86] M. Narasimhan, D. Patel, D. Vedpathak, M. Rathinam, G. Henderson, and L. Mahimainathan, "Identification of novel microRNAs in post-transcriptional control of Nrf2 expression and redox homeostasis in neuronal, SH-SY5Y cells," PLoS One, vol. 7, no. 12, article e51111, 2012.

[87] B. Wang, Y. Teng, and Q. Liu, "MicroRNA-153 regulates NRF2 expression and is associated with breast carcinogenesis," Clinical Laboratory, vol. 62, no. 1-2, pp. 39-47, 2016.

[88] Y. Jing and B. Xu, "Decreased expression of miR-153-3p in oral squamous cell carcinoma contributes to cell migration and invasion," International Journal of Clinical and Experimental Pathology, vol. 10, no. 5, pp. 6117-6129, 2017.

[89] L. Shi, Z. G. Chen, L. L. Wu et al., "miR-340 reverses cisplatin resistance of hepatocellular carcinoma cell lines by targeting Nrf2-dependent antioxidant pathway," Asian Pacific Journal of Cancer Prevention, vol. 15, no. 23, pp. 10439-10444, 2014.

[90] M. Qaisiya, C. D. Coda Zabetta, C. Bellarosa, and C. Tiribelli, "Bilirubin mediated oxidative stress involves antioxidant response activation via Nrf2 pathway," Cellular Signalling, vol. 26, no. 3, pp. 512-520, 2014.

[91] S. Yamamoto, J. Inoue, T. Kawano, K. Kozaki, K. Omura, and J. Inazawa, "The impact of miRNA-based molecular diagnostics and treatment of NRF2-stabilized tumors," Molecular Cancer Research, vol. 12, no. 1, pp. 58-68, 2014.

[92] M. T. M. Van Jaarsveld, J. Helleman, A. W. M. Boersma et al., "miR-141 regulates KEAP1 and modulates cisplatin sensitivity in ovarian cancer cells," Oncogene, vol. 32, no. 36, pp. 4284-4293, 2013.

[93] L. Shi, L. Wu, Z. Chen et al., "MiR-141 activates Nrf2dependent antioxidant pathway via down-regulating the expression of Keap1 conferring the resistance of hepatocellular carcinoma cells to 5-fluorouracil," Cellular Physiology and Biochemistry, vol. 35, no. 6, pp. 2333-2348, 2015.

[94] G. Eades, Y. Yao, M. Yang, Y. Zhang, S. Chumsri, and Q. Zhou, "miR-200a regulates SIRT1 expression and epithelial to mesenchymal transition (EMT)-like transformation in mammary epithelial cells," The Journal of Biological Chemistry, vol. 286, no. 29, pp. 25992-26002, 2011.

[95] M. Liu, C. Hu, Q. Xu et al., "Methylseleninic acid activates Keap1/Nrf2 pathway via up-regulating $m i R-200 a$ in human oesophageal squamous cell carcinoma cells," Bioscience Reports, vol. 35, no. 5, article e00256, 2015.

[96] S. Kabaria, D. C. Choi, A. D. Chaudhuri, M. R. Jain, H. Li, and E. Junn, "MicroRNA-7 activates Nrf2 pathway by targeting Keap1 expression," Free Radical Biology \& Medicine, vol. 89, pp. 548-556, 2015.

[97] W. Hou, Q. Tian, J. Zheng, and H. L. Bonkovsky, "MicroRNA-196 represses Bach1 protein and hepatitis $C$ virus gene expression in human hepatoma cells expressing hepatitis $\mathrm{C}$ viral proteins," Hepatology, vol. 51, no. 5, pp. 1494-1504, 2010.

[98] B. Akdemir, Y. Nakajima, J. Inazawa, and J. Inoue, " $m i R-432$ induces NRF2 stabilization by directly targeting KEAP1," Molecular Cancer Research, vol. 15, no. 11, pp. 1570-1578, 2017. 
[99] S. Tokumaru, M. Suzuki, H. Yamada, M. Nagino, and T. Takahashi, "let-7 regulates Dicer expression and constitutes a negative feedback loop," Carcinogenesis, vol. 29, no. 11, pp. 2073-2077, 2008.

[100] C. D. Johnson, A. Esquela-Kerscher, G. Stefani et al., "The let-7 microRNA represses cell proliferation pathways in human cells," Cancer Research, vol. 67, no. 16, pp. 77137722, 2007.

[101] S. M. Johnson, H. Grosshans, J. Shingara et al., "RAS is regulated by the let-7 microRNA family," Cell, vol. 120, no. 5, pp. 635-647, 2005.

[102] J. F. Reichard, G. T. Motz, and A. Puga, "Heme oxygenase-1 induction by NRF2 requires inactivation of the transcriptional repressor BACH1," Nucleic Acids Research, vol. 35, no. 21, pp. 7074-7086, 2007.

[103] S. Li, T. Chen, Z. Zhong, Y. Wang, Y. Li, and X. Zhao, "microRNA-155 silencing inhibits proliferation and migration and induces apoptosis by upregulating $\mathrm{BACH} 1$ in renal cancer cells," Molecular Medicine Reports, vol. 5, no. 4, pp. 949-954, 2012.

[104] W. Hou, Q. Tian, N. M. Steuerwald, L. W. Schrum, and H. L. Bonkovsky, "The let-7 microRNA enhances heme oxygenase1 by suppressing Bach1 and attenuates oxidant injury in human hepatocytes," Biochimica et Biophysica Acta (BBA) Gene Regulatory Mechanisms, vol. 1819, no. 11-12, pp. 1113-1122, 2012.

[105] S. Sati, S. Ghosh, V. Jain, V. Scaria, and S. Sengupta, "Genome-wide analysis reveals distinct patterns of epigenetic features in long non-coding RNA loci," Nucleic Acids Research, vol. 40, no. 20, pp. 10018-10031, 2012.

[106] V. A. Moran, R. J. Perera, and A. M. Khalil, "Emerging functional and mechanistic paradigms of mammalian long non-coding RNAs," Nucleic Acids Research, vol. 40, no. 14, pp. 6391-6400, 2012.

[107] N. M. White, C. R. Cabanski, J. M. Silva-Fisher, H. X. Dang, R. Govindan, and C. A. Maher, "Transcriptome sequencing reveals altered long intergenic non-coding RNAs in lung cancer," Genome Biology, vol. 15, no. 8, p. 429, 2014.

[108] P. Thai, S. Statt, C. H. Chen, E. Liang, C. Campbell, and $\mathrm{R}$. Wu, "Characterization of a novel long noncoding RNA, SCAL1, induced by cigarette smoke and elevated in lung cancer cell lines," American Journal of Respiratory Cell and Molecular Biology, vol. 49, no. 2, pp. 204-211, 2013.

[109] Y. Zhang, J. Xia, Q. Li et al., "NRF2/long noncoding RNA ROR signaling regulates mammary stem cell expansion and protects against estrogen genotoxicity," The Journal of Biological Chemistry, vol. 289, no. 45, pp. 31310-31318, 2014. 


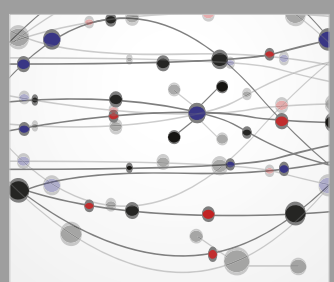

The Scientific World Journal
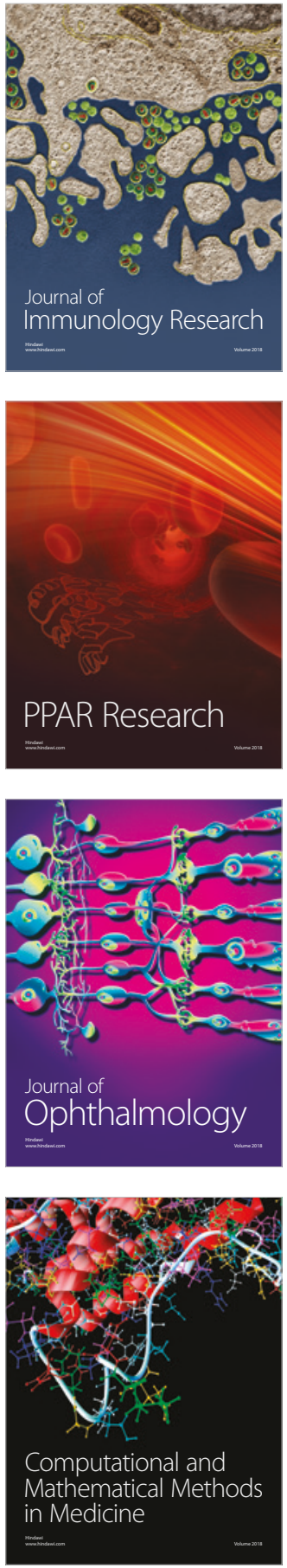

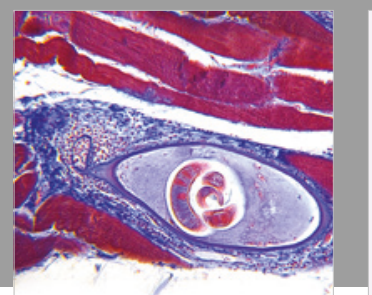

Gastroenterology Research and Practice

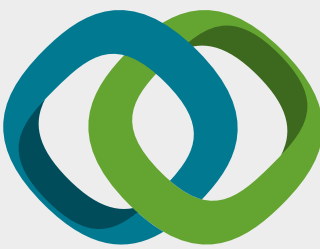

\section{Hindawi}

Submit your manuscripts at

www.hindawi.com
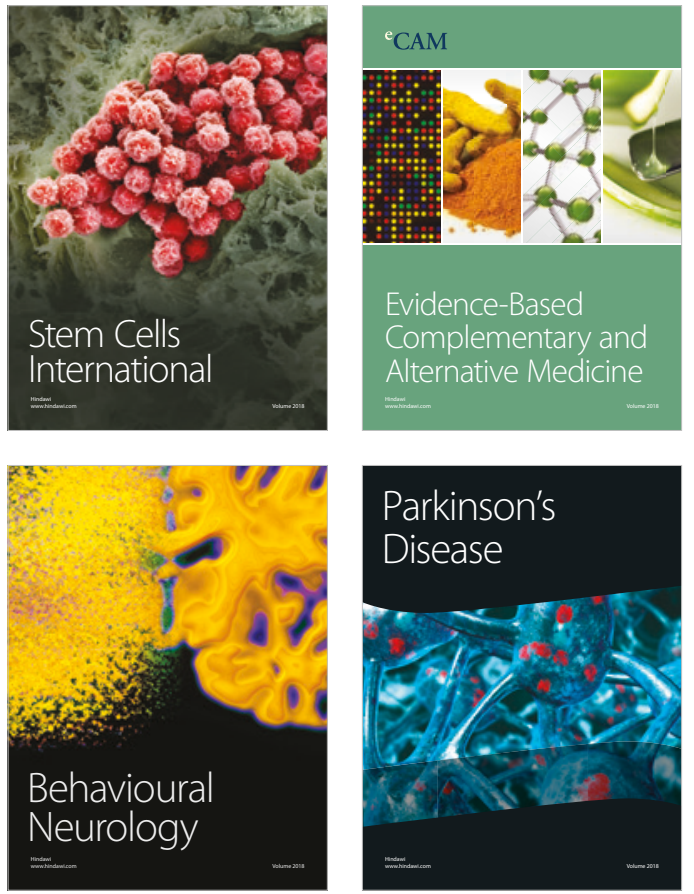

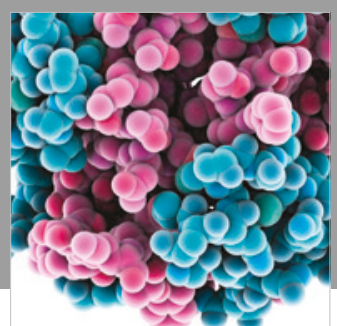

ournal of

Diabetes Research

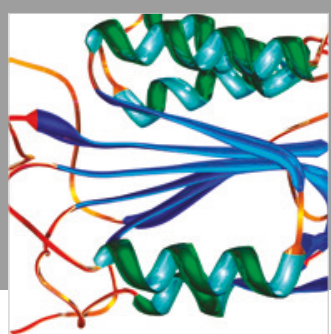

Disease Markers
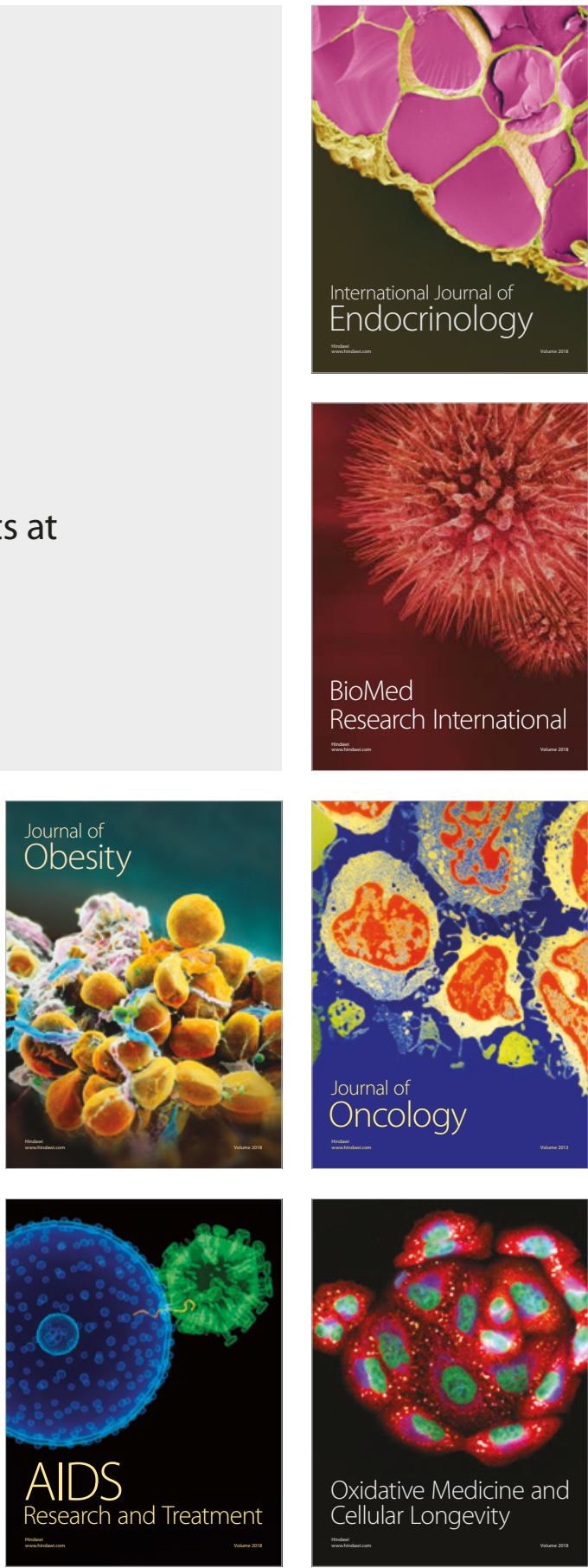